\title{
Bayesian Detection of Changepoints in Finite-State Markov Chains for Multiple Sequences
}

\section{Petter Arnesen, Tracy Holsclaw and Padhraic Smyth 『}

We consider the analysis of sets of categorical sequences consisting of piecewise homogeneous Markov segments. The sequences are assumed to be governed by a common underlying process with segments occurring in the same order for each sequence. Segments are defined by a set of unobserved changepoints where the positions and number of changepoints can vary from sequence to sequence. We propose a Bayesian framework for analyzing such data, placing priors on the locations of the changepoints and on the transition matrices and using Markov chain Monte Carlo (MCMC) techniques to obtain posterior samples given the data. Experimental results using simulated data illustrates how the methodology can be used for inference of posterior distributions for parameters and changepoints, as well as the ability to handle considerable variability in the locations of the changepoints across different sequences. We also investigate the application of the approach to sequential data from two applications involving monsoonal rainfall patterns and branching patterns in trees.

Key words: Changepoint model; Cross-validation; Hidden Markov model; Multiple sequences.

\section{INTRODUCTION}

Finite-state Markov chains are widely used to model sequential data in applications such as weather models (Gabriel and Neumann 1962), speech recognition (Rabiner 1989), bioinformatics (Durbin et al. 1998), and more (Guttorp 1995). A common assumption is that the chain is homogeneous, often motivated by a desire to keep the number of model parameters tractable. In practice, however, inhomogeneity in various forms is often present.

In particular in this paper we investigate the problem of modeling sets of categorical-valued sequences where each sequence is assumed to be generated by an ordered set of segments, with

Petter Arnesen is a PhD student with the Department of Mathematical Sciences, Norwegian University of Science and Technology, Trondheim 7491, Norway (email: petterar@math.ntnu.no). Tracy Holsclaw is a Postdoctoral Scholar with the Department of Statistics, University of California, Irvine, CA (email: tholscla@ams.ucsc.edu). Padhraic Smyth is a Professor with the Department of Computer Science and the Department of Statistics, University of California, Irvine, CA (email: smyth@ics.uci.edu). 


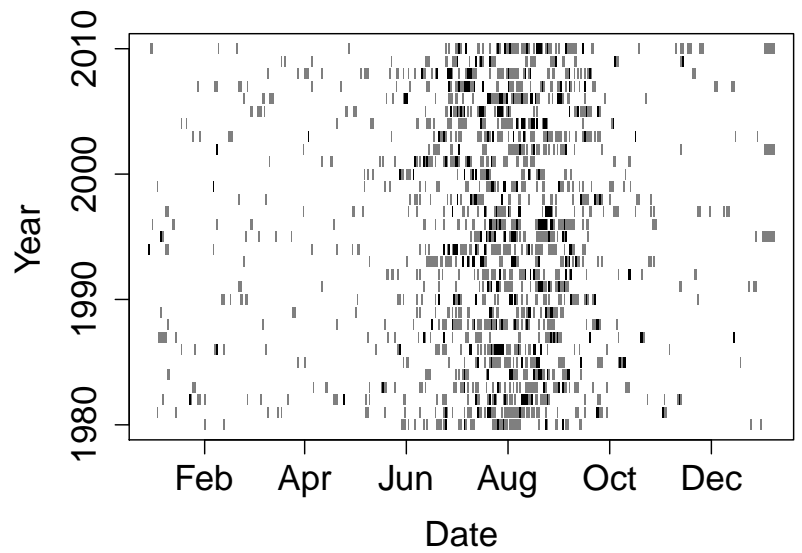

Figure 1: 31 years of rainfall categorized into no/light rainfall (white), medium rainfall (gray), and heavy rainfall (black).

unobserved segment boundaries that can vary from sequence to sequence. Each segment has its own Markov dynamics, representing common "phases" for some underlying process. As a specific example, consider the modeling of rainfall at a particular location. Markov chains have a long history of use as stochastic models of rainfall (Newnham 1916; Gold 1929; Cochran 1938; Wilks and Wilby 1999; Chen et al. 2010). Figure 1 is an illustration of annual daily rainfall sequences for a weather station in Northern India. The appearance of the Indian monsoon in early summer causes a visible phase-change in the sequence of daily rainfall for each year, motivating the type of segment-based model that is the focus of this paper. Being able to infer monsoon onset and withdrawal dates, and to effectively handle interannual variability, is of considerable interest in agriculture modeling and climate science (e.g., Joseph et al. (1994)) . Other examples of data sets characterized by multiple sequences with common segmental structure include tree branching patterns (Guédon et al.|2001), sequences of bird songs (Craig 1943; Rafterv and Tavaré 1994), wind-speed data (Berchtold 1999), DNA sequences (Berchtold 2002; Fearnhead and Liu 2007), and dialog transcripts (Levin and Pieraccini (2000).

In this paper we model a particular type of shared structure where a categorical sequence is assumed to consist of $K+1$ segments separated by $K$ unobserved changepoints. Within each segment the observed data are assumed to be generated by a homogeneous finite-state 
Markov chain. We will assume that each of the $K+1$ segments has its own Markov parameters, resulting in $K+1$ transition matrices. The location of each changepoint is modeled by a discrete distribution conditioned on the location of the previous changepoint. We will consider the case of a set of $L$ observed sequences (of potentially varying length) where the locations of the $K$ changepoints can vary across the sequences. The $L$ sequences share information in that the parameters of the Markov transition matrices are global, and the order in which the segments appear in each sequence are assumed to be the same, corresponding to common underlying phases. Our framework allows any segment to be skipped in a particular sequence, and thus, $K$ is in effect the maximal number of changepoints that can occur in any particular sequence.

There is a large amount of prior literature on changepoint detection for sequential data, covering a variety of aspects of this problem, including online/offline estimation, single versus multiple sequences, Bayesian versus non-Bayesian inference, and methods for inferring the number of changepoints. Much of this prior work has focused on the case of independent observations and/or non-categorical data. An exception is the double-Markov chain approach proposed by Berchtold (1999) using the Expectation-Maximization (EM) algorithm for inference, and which was further developed within a Bayesian framework by Fitzpatrick and Marchev (2013). Because of the Markov assumption at the segment-transition level, the model imposes an implicit geometric distribution on segment lengths which is not always ideal in practice (we will followup on this point later in the paper). The geometric assumption can be relaxed by using a hidden semi-Markov approach for example (Guédon 2003), but this imposes computational restrictions, namely that the time complexity of inference (e.g., via the forward-backward algorithm, Rabiner (1989)) can scale as $O\left(T^{2}\right)$ where $T$ is the length of a sequence.

Polansky (2007) proposed a likelihood-based framework for segmentation assuming a process that switches between different Markov chains with unknown parameters, but for the 
case of a single sequence rather than multiple sequences. A restricted variant of the multiple sequence changepoint problem is the case where sequences are required to be of the same length and the changepoints are assumed to occur at the same location in each sequence, which can also be viewed as a single multivariate sequence (Lai and Xing 2011; Xing et al. 2012; Fitzpatrick and Marchev 2013). Zhang and Siegmund (2012) relax this approach to allow subsets of sequences (of equal length and for the case of independent real-valued observations) to share changepoints. In the approach proposed here we allow each sequence to have its own changepoint locations and sequences to have different lengths.

While most prior work has focused on likelihood-based inference and point estimates of changepoints, here we use full Bayesian inference for both changepoint locations and model parameters. Our MCMC inference algorithm has a time complexity per iteration that is linear in the length $T$ of a sequence, avoiding the $O\left(T^{2}\right)$ time complexity incurred by Rigaill et al. (2012). Fearnhead and Liu (2011) developed a Bayesian changepoint detection that is linear in $T$ in terms of time complexity, but for the case of a single real-valued sequence.

Our focus on multiple sequences also provides a natural context for using cross-validation (across sequences) for model selection, providing a practical alternative to approaches such as the BIC criterion (Guédon 2003; Polansky 2007; Fitzpatrick and Marchev 2013; Luong et al. 2013), and related penalized likelihood approaches (Zhang and Siegmund 2007; Rigaill et al. 2012; Cleynen and Lebarbier 2013), which are not always appropriate or effective for changepoint problems (e.g., see the discussion in Cleynen et al. (2014)).

In Section 2 we introduce our proposed changepoint Markov model, with results on model selection in Section 3. In Section 4.1 we present an example of simulated data to illustrate different aspects of the proposed approach. Section 4.2 describes the application of our approach to a data set of annual branching of apple trees, and in Section 4.3 we discuss the application 
of the model to a data set consisting of multiple years of daily rainfall in India. We conclude the paper in Section 5 with a brief discussion of the main results as well as suggestions for future directions. Discussions on MCMC sampling, how to handle missing data and additional simulated examples are provided in the supplementary materials.

\section{A CHANGEPOINT MARKOV MODEL FOR CATEGORICAL SEQUENCES}

In this section we define our Bayesian model for detection of a fixed number of changepoints in multiple sequences. The MCMC sampling algorithm we construct for sampling from the resulting posterior distribution is given in Section S.1 in the supplementary materials.

\subsection{Homogeneous Markov Chains}

Given a sequence of discrete observations $\mathbf{y}=\left(y_{1}, \ldots, y_{T}\right)$, such that $y_{j} \in\{1, \ldots, n\}$ for all $j=1, \ldots, T, \mathbf{y}$ is a realization from a finite-state Markov chain if the joint probability of $\mathbf{y}$ can be written as

$$
p(\mathbf{y})=p_{1}\left(y_{1}\right) \prod_{j=2}^{T} p_{j}\left(y_{j} \mid y_{j-1}, \ldots, y_{1}\right)=\prod_{j=1}^{T} p_{j}\left(y_{j} \mid y_{j-1}\right),
$$

where the conditional distribution of $y_{j}$ given $y_{j-1}, \ldots, y_{1}$ only depends on the previous state $y_{j-1}$, and we assume some given initial state $y_{0}$ such that $p_{1}\left(y_{1}\right)=p_{1}\left(y_{1} \mid y_{0}\right)$. If the transition distribution $p_{j}\left(y_{j} \mid y_{j-1}\right)=p\left(y_{j} \mid y_{j-1}\right)$ for all $j=1, \ldots, T$, we say that the Markov chain is homogeneous. The transition probabilities can be organized into an $n \times n$ transition matrix $\mathbf{Q}$, where the rows indicate the value $y_{j-1}$ and the columns represent the values of $y_{j}$. We relax these assumptions below to allow the Markov chain to be piecewise homogeneous, allowing inhomogeneity via local segmentation of the sequence $\boldsymbol{y}$. 


\subsection{Modeling Local Segments}

To define a piecewise homogeneous Markov chain, we divide the $T$ observations into $K+1$ segments by introducing $K$ changepoints $\tau_{0}=0 \leq \tau_{1} \leq \ldots \leq \tau_{K} \leq T$, and write $\boldsymbol{\tau}=\left(\tau_{1}, \ldots, \tau_{K}\right)$ as the vector of changepoints. If $\tau_{i-1}<\tau_{i}$ then we denote $s_{i}=\left\{\tau_{i-1}+1, \ldots, \tau_{i}\right\}$ to be the $i^{t h}$ segment and if $\tau_{i-1}=\tau_{i}$ (a segment of length zero) then $s_{i}=\emptyset$. Introducing the notation $\boldsymbol{y}_{s}=\left(y_{j} \mid j \in s\right)$ for $s \subseteq\{1, \ldots, T\}$, the $i^{\text {th }}$ segment will include the data points $\boldsymbol{y}_{s_{i}}$. We assume the locations of the $K$ changepoints to be generated by a distribution of the form

$$
p(\boldsymbol{\tau} \mid T, \overline{\boldsymbol{\theta}})=p\left(\tau_{1}, \ldots, \tau_{K} \mid T, \overline{\boldsymbol{\theta}}\right)=\prod_{i=1}^{K} p\left(\tau_{i} \mid \tau_{i-1}, T, \boldsymbol{\theta}_{i}\right)
$$

where $p\left(\tau_{i} \mid \tau_{i-1}, T, \boldsymbol{\theta}_{i}\right)$ is a discrete parametric distribution for changepoint $\tau_{i}$, with parameter vector $\boldsymbol{\theta}_{i}$, and where we let $\overline{\boldsymbol{\theta}}$ denote the collection of the parameter vectors $\boldsymbol{\theta}_{i}$ for $i=1, \ldots, K$. In the remainder of the paper we assume the distribution for $\tau_{i}$ to be the negative binomial distribution truncated to the interval $\left(\tau_{i-1}, T\right)$ (additional details in Section 2.4). The twoparameter negative binomial distribution provides additional flexibility in modeling segment lengths compared to a single parameter distribution such as the geometric distribution. This can lead to more accurate detection of changepoints, as we will see later in the paper.

\subsection{The Piecewise Homogeneous Markov Chain}

We assume that within each segment the sequential observations in $\boldsymbol{y}$ evolve according to a fixed transition matrix, i.e., $\boldsymbol{y}$ is generated by a piecewise homogeneous Markov chain within each segment. With $K$ changepoints, we have a total of $K+1$ transition matrices $\mathbf{Q}^{(1)}, \ldots, \mathbf{Q}^{(K+1)}$, each having size $n \times n$. In what follows we present the case where the $K+1$ transition matrices are modeled separately and independently, but it is straightforward to constrain some of these 
matrices to be the same (as in our rainfall example later in the paper) and to reduce the parameter count accordingly. Let $\overline{\mathbf{Q}}$ denote the collection of these $K+1$ transition matrices, and let $\mathbf{Q}_{k, l}^{(i)}$ denote the element at the $k^{t h}$ row and the $l^{\text {th }}$ column of matrix $i$. Given an initial state $y_{0}$, the data likelihood is

$$
p\left(\mathbf{y} \mid \boldsymbol{\tau}, \overline{\mathbf{Q}}, y_{0}\right)=\prod_{j=1}^{T} p\left(y_{j} \mid y_{j-1}, \overline{\mathbf{Q}}, \boldsymbol{\tau}\right)=\prod_{i=1}^{K+1} \prod_{j \in s_{i}} \mathbf{Q}_{y_{j-1}, y_{j}}^{(i)}
$$

Assume for the moment that no segments are of length zero, i.e., no segments are skipped. If $y_{j}$ is the first data point in segment $s_{i}, i>1$, then we assume it to be distributed according to the conditional distribution of $y_{j}$ given $y_{j-1}$ (the last point in segment $s_{i-1}$ ) using the transition matrix for segment $s_{i}, \mathbf{Q}^{(i)}$. The extension to segments of length zero is straightforward.

We adopt a fully Bayesian approach conditioned on fixed $K$ for our model. For priors for the transition matrices we assume each of the rows to be independently distributed according to the Dirichlet distribution. In particular, $\mathbf{Q}_{k, \cdot}^{(i)} \sim \operatorname{Dir}\left(\boldsymbol{\alpha}_{k}^{(i)}\right)$, where $\mathbf{Q}_{k, \cdot}^{(i)}$ is the $k^{\text {th }}$ row of the $i^{t h}$ transition matrix and where $\operatorname{Dir}\left(\boldsymbol{\alpha}_{k}^{(i)}\right)$ denotes the Dirichlet distribution with parameter vector $\boldsymbol{\alpha}_{k}^{(i)}$ of appropriate length. We set all elements in $\boldsymbol{\alpha}_{k}^{(i)}$ to be equal to 1 , for all $i$ and $k$ in our examples, rendering the prior equivalent to having seen one transition from each category to every other category including itself.

\subsection{Modeling Multiple Sequences of Variable Length}

To handle multiple sequences, consider $L$ conditionally independent sequences of observations, $\boldsymbol{y}^{(l)}, l=1, \ldots, L$, with lengths $T_{1}, \ldots, T_{L}$, where each sequence consists of $K+1$ segments occurring in the same order as described above. Also let $y_{0}^{(l)}$ denote the initial value for each of the $l$ sequences. Assuming the sequences to be conditionally independent, the likelihood for 
multiple sequences is the product of the likelihoods for each individual sequence (as defined earlier). Segments are allowed to be of zero length (i.e., skipped), effectively allowing the number of changepoints per sequence to differ (see the simulation studies in Section S.3.1 and S.3.2 in the supplementary material for examples of this property). Let $\boldsymbol{\tau}^{(l)}=\left(\tau_{1}^{(l)}, \ldots, \tau_{K}^{(l)}\right)$ denote the changepoints in sequence $l$. There are a number of options for modeling how the locations of the changepoints $\boldsymbol{\tau}^{(l)}$ are related to the lengths of the sequences $T_{l}$. One could allow the changepoints to have distributions defined in absolute units (e.g., of time) and treat the total length of the sequence as a random quantity. The approach we take here is to assume that the distribution on changepoints (or equivalently, on segment length) is specified in terms of position relative to the total length of the sequence, where we treat the observed total sequence lengths $T_{l}$ as fixed quantities and condition on them. In practice the choice of parametrization will depend on the specific nature of the application, and for the special case where the sequences are all of the same length, the absolute and relative approaches will be equivalent. In particular we assume that the position of the changepoints $\tau_{i}^{(l)}$ have a negative binomial distribution, with parameters $\boldsymbol{\theta}_{i}=\left(r_{i}, b_{i}\right) \in(0,1) \times(0,1)$, truncated to the range $\left(\tau_{i-1}^{(l)}, T_{l}\right)$. We write

$$
p\left(\tau_{i}^{(l)} \mid \tau_{i-1}^{(l)}, T_{l}, \boldsymbol{\theta}_{\boldsymbol{i}}\right) \propto \frac{\Gamma\left(\tau_{i}^{(l)}+\gamma\left(\boldsymbol{\theta}_{i}, T_{l}\right)\right)}{\tau_{i}^{(l)} ! \Gamma\left(\gamma\left(\boldsymbol{\theta}_{i}, T_{l}\right)\right)} b_{i}^{\gamma\left(\boldsymbol{\theta}_{i}, T_{l}\right)}\left(1-b_{i}\right)^{\tau_{i}^{(l)}}, \tau_{i}^{(l)}=\tau_{i-1}^{(l)}, \ldots, T_{l},
$$

where $\gamma\left(\boldsymbol{\theta}_{i}, T_{l}\right)$ is defined via the expression $r_{i} T_{l}=\gamma\left(\boldsymbol{\theta}_{i}, T_{l}\right)\left(1-b_{i}\right) / b_{i}$, which corresponds to the expected value of the negative binomial distribution without truncation. The parameter $r_{i}$ will therefore be related to the expected position of changepoint $\tau_{i}^{(l)}$ scaled by the length $T_{l}$ of sequence $l$, while $b_{i}$ will be related to variance of the distribution. This truncated distribution can be efficiently computed as it does not include a computationally demanding normalizing constant. We also assume apriori that the $\boldsymbol{\theta}_{i}, i=1, \ldots, K$ are independent, and that $r_{i}$ and $b_{i}$ are independent and uniformly distributed in the unit interval, $U(0,1)$. 


\section{MODEL SELECTION AND COMPARISON}

Previous work on estimating the number of changepoints has often used formulations such as BIC (Guédon 2003; Polansky 2007; Fitzpatrick and Marchev 2013; Luong et al. 2013). The BIC criterion, however, is not directly applicable to changepoint problems (as discussed in Zhang and Siegmund (2007)), leading to alternative penalized likelihood formulations, see for instance Zhang and Siegmund (2007) Cleynen and Lebarbier (2013) and Clevnen et al. (2014). More fully Bayesian approaches have also been pursued (for example, by Fearnhead and Liu (2011) and Rigaill et al. (2012)) but for single-sequences with real or count-valued IID observations.

For multiple sequences, Xing et al. (2012) investigate a Bayesian approach for inferring boundaries for piecewise homogeneous Markov chains with unobserved changepoints, similar to the problem we address in this paper. However, their approach assumes that all sequences are the same length and have changepoints in the same position, essentially restricting the approach to the case of a single sequence with a multivariate distribution. For IID observations Zhang and Siegmund (2012) also consider the scenario of multiple equi-length sequences with aligned changepoints across sequences, using a modified BIC criterion for model selection.

An alternative approach that could be pursued is that of transdimensional MCMC algorithms based on a joint posterior distribution for the model and parameter space (Green 1995). Reversible jump MCMC (RJMCMC) algorithms (Green 1995) could be used in this context, but we would anticipate slow convergence and potential sensitivity issues when choosing proposal distributions (particularly for the jump proposals). Another option is the Dirichlet process framework, although it would not be straightforward to apply this approach given the computational challenges that would result from the lack of conjugacy (Neal 2000). 
Given these various issues with Bayesian and information-based criteria (at least in the context of our proposed model), we instead use cross-validation with log-probability scores (e.g., see Vehtari and Lampinen (2002); Gneiting and Raftery (2007); Gelman et al. (2014); Li et al. (2014)) for both choosing the number of changepoints and for model selection among alternative frameworks. We take advantage of the fact that we are working with multiple sequences and use cross-validation at the sequence level, using the log-probability of held-out sequences as our scoring function. Our simulation results (next section) suggest that this approach is feasible even with a relatively small number of sequences (e.g., 10). When training our model, we hold out $t$ of our $L$ sequences, referred to as the test set, and train our model using only $L-t$ of the available sequences, referred to as the training set. Denote the $L-t$ sequences in a training set by $\boldsymbol{Y}_{D}$ and the $t$ sequences in the test set by $\boldsymbol{Y}_{-D}$. To evaluate the quality of the model, we estimate the logarithm of the probability of observing the test set $\boldsymbol{Y}_{-D}$ given the training set $\boldsymbol{Y}_{D}$ and the model. We average this log-probability for a number of different train/test set pairs using cross-validation. The Monte Carlo estimate of the log-probability of one of the sequences $\boldsymbol{y}^{(l)}$ in the test set is

$$
\ln p\left(\boldsymbol{y}^{(l)} \mid \boldsymbol{Y}_{D}\right)=\frac{1}{N} \sum_{i=1}^{N} \frac{1}{M} \sum_{j=1}^{M} \ln p\left(\boldsymbol{y}^{(l)} \mid \boldsymbol{\tau}^{(l)[j]}, \overline{\mathbf{Q}}^{[i]}, y_{0}^{(l)}\right)
$$

where $\boldsymbol{\tau}^{(l)[j]} \sim p\left(\boldsymbol{\tau}^{(l)} \mid T_{l}, \overline{\boldsymbol{\theta}}^{[i]}\right)$ for $j=1, \ldots, M$, and $\left(\overline{\boldsymbol{\theta}}^{[i]}, \overline{\mathbf{Q}}^{[i]}\right) \sim p\left(\overline{\boldsymbol{\theta}}, \overline{\mathbf{Q}} \mid \boldsymbol{Y}_{D}\right)$ for $i=1, \ldots, N$, and where we use superscript [.] to denote simulated samples. After convergence of the algorithm and for $N$ independent samples of $\overline{\boldsymbol{\theta}}$ and $\overline{\mathbf{Q}}$, we simulate $M$ samples from $p\left(\boldsymbol{\tau}^{(l)} \mid T_{l}, \overline{\boldsymbol{\theta}}\right)$. For each of these $M$ samples, we calculate the value of the logarithm of the data likelihood in (3), and then compute the mean. For the results in this paper we used $M=1000$ samples, although sensitivity analysis (not shown) indicates that $M=100$ is sufficient to obtain consistent estimates of the log-probability score. For $N$, which is the number of independent samples from the MCMC 
algorithm we used $N=1000$ following the discussion in Section S.1 in the supplementary materials on burn-in period and thinning. For all of our results we use 10-fold cross-validation, i.e. we partition our data into 10 test and training sets (unless otherwise stated).

The total log-probability score is defined to be the sum of the individual scores divided by the sum of the length of the sequences

$$
S\left(\boldsymbol{Y}_{-D} \mid \boldsymbol{Y}_{D}\right)=\frac{\sum_{l=1}^{t} \ln p\left(\boldsymbol{y}^{(l)} \mid \boldsymbol{Y}_{D}\right)}{\sum_{l=1}^{t} T_{l}}
$$

where $\boldsymbol{y}^{(1)}, \ldots, \boldsymbol{y}^{(t)}$ are the sequences in the test set. Using the equation above we can compare different models, either our proposed model with different numbers of changepoints, or our proposed model versus an alternative model. When calculating the cross-validated log-probability scores for two models $\mathcal{M}_{1}$ and $\mathcal{M}_{2}$ we use the same train/test sets and report the difference between the scores for each train/test set, i.e., we calculate $S\left(\boldsymbol{Y}_{-D} \mid \boldsymbol{Y}_{D}, \mathcal{M}_{1}\right)-S\left(\boldsymbol{Y}_{-D} \mid \boldsymbol{Y}_{D}, \mathcal{M}_{2}\right)$ for each train/test set.

\section{ANALYSIS AND RESULTS}

In this section, we analyze one simulated and two real data sets. For the simulated data we generate the sequences and changepoints, allowing us to test our model and compare to alternative methods. Additional simulated data sets are provided in the supplementary material to further explore different aspects of our model. The two real data sets consists of branching patterns for apple trees and daily rainfall over a region Northern India.

In all of our analyses we compare our method to three baseline models. The first baseline SI (Segmental-Independence) is the same as our proposed model but assumes the observations $\boldsymbol{y}$ are independent within each segment. The second baseline is a standard hidden Markov model 
(HMM) with a left-to-right transition matrix. The third baseline is the double hidden Markov chain model (dHMM) from Berchtold (1999) with a left-to-right transition matrix. These three baselines differ from our proposed model in that the SI and HMM models assume independence of the observations $\boldsymbol{y}$ (rather than Markov dependence), and the HMM and dHMM models assume geometric distributions on the changepoint locations (rather than a negative-binomial distribution). For each of the three baselines we use a Bayesian inference procedure similar to that described in Section S.1 in the supplementary materials, and we report the results for the models with the number of changepoints corresponding to the highest log-probability score (which for all the baselines turned out to be the same number of changepoints as in our preferred model). In addition to comparing our model to these baseline models we also compare our results to those obtained with a model where each sequence is analyzed independently. In

particular, we use the single-sequence model of Fearnhead (2006) for comparison, originally proposed for real-valued data with independence. See the supplementary materials for these results.

\subsection{Synthetic Data Example: Scenario 1}

We simulated $L=10$ binary sequences, all of length 200, from our proposed model. For each sequence $l=1, \ldots, 10$, we simulated one changepoint $\tau_{1}^{(l)}$, and the position of the changepoint was generated from the truncated negative binomial distribution as explained in Section 2.4. using the parameters $r_{1}=0.5$ and $b_{1}=0.8$, such that each segment will have length about 100 . The transition matrices used to generate the binary data in each segment had diagonal entries $q_{1, i}$ and $q_{2, i}$, with $i$ denoting the segment. We used $q_{1,1}=q_{2,1}=0.8$ in the first segment, and $q_{1,2}=0.5$ and $q_{2,2}=0.4$ in the second segment.

We used our cross-validation model selection procedure described in Section 3 to determine 


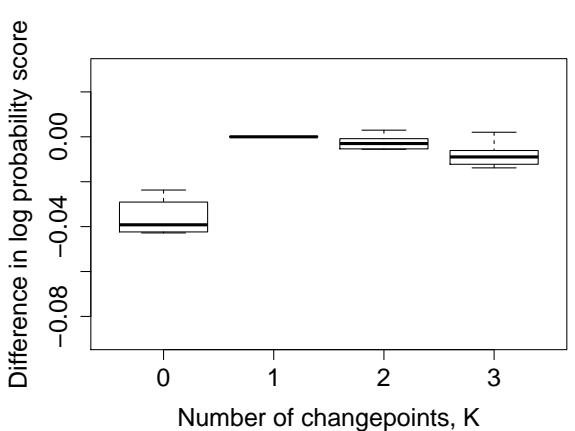

(a)

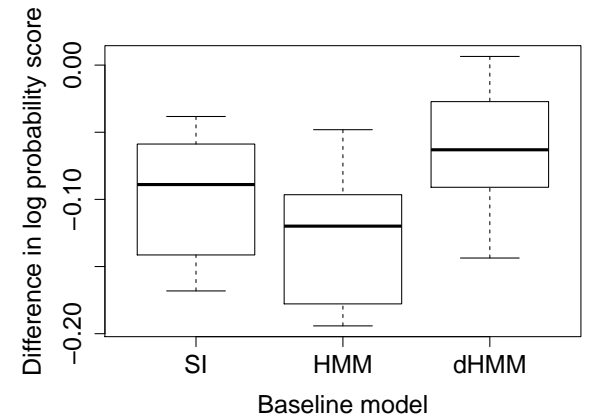

(b)

Figure 2: Each boxplot shows the log-probability scores, across the validation sets, for a different model. The y-axis is defined as the log-probability score for (a) other numbers of changepoints and (b) other baseline models, minus the log-probability score for the model with one changepoint.

the number of changepoints. We defined 10 train/test sets by leaving out one of the 10 sequences in each fold, resulting in a "hold-one-sequence-out" cross-validation test. The result of this model comparison is shown in Figure 2(a). It is clear from the figure that the model with a single changepoint $(K=1)$ is the preferred one. As the number of changepoints increases beyond a single changepoint the cross-validated log-probability scores become slightly worse.

The cross-validated log-probability scores of the three baseline models relative to our model are shown in Figure 2(b), and as we can see our model performs the best. All models were fit using the optimal changepoint number $(K=1)$.

We then trained our model with the correct number of changepoints $(K=1)$, using all 10 sequences. The estimated parameter values with $95 \%$ credible intervals (CI) (corresponding to the $2.5 \%$ maximum and minimum percentiles of the posterior samples after convergence) are shown in Table S.1 and Table S.2 in the supplementary materials. All of the parameters are well estimated, although there is considerable uncertainty concerning the estimated value of $b=b_{1}$. For a more detailed discussion of the posterior analysis for $b$ and $r$ see the supplementary materials Section S.2.1.

Figure 3(a) shows, for each sequence, the estimated marginal probability that each obser- 


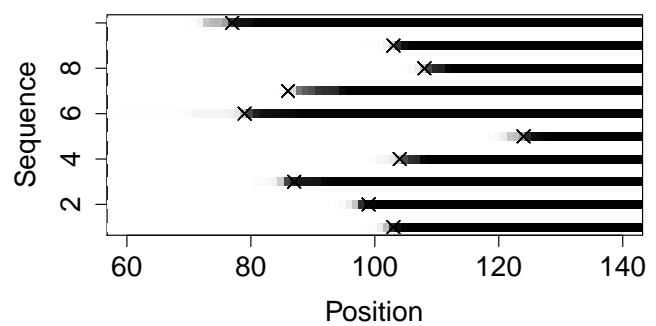

(a)

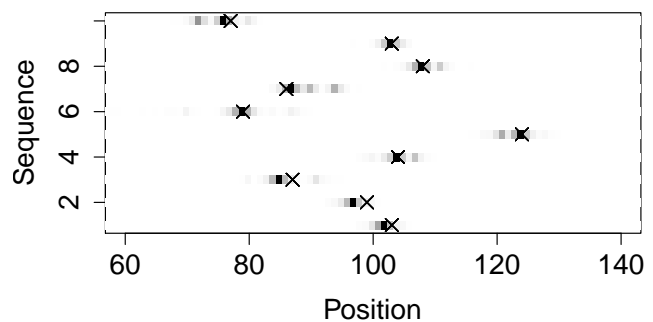

(b)

Figure 3: The estimated marginal probabilities of (a) the classification of each observation to segment 2 (probability 1 is black) and (b) a particular position being a changepoint, where the gray scale has been adjusted for each sequence so that the position with the maximum probability is black and the position with the minimum probability is white. The true changepoint locations are marked with $(\times)$ in both plots. Note that only position 60 to 140 are shown in each sequence.

vation in that sequence belongs to segment 2 . The true changepoint is marked (with an $\times$ ) for each sequence and, as we can see, the changepoints are well recovered. In Figure 3(b), we see the marginal probability plot for the positions of the changepoints. It is worth commenting on the fact that the marginal probabilities in Figure 3(b) do not necessarily vary smoothly as a function of location. That is, there are observations that are considered to be unlikely candidates for a changepoint even though the previous and next observations have a high probability of being a changepoint.

\subsection{Real Data Analysis: Branching of Apple Trees}

In this section we analyze the apple tree branching data sett presented in Guédon et al. (2001) and Guédon (2003). Guédon et al. (2001) describe how the branching structure of the first annual shoot of the trunk can be useful as a predictor of the adult tree structure and more broadly how branching sequences play an important role in understanding plant development.

${ }^{1}$ The data set is available as part of the AMAPmod software (Godin et al. 1997) available at http://openalea.gforge.inria.fr/dokuwiki/doku.php. For more details the reader is referred to Godin et al. (1999) 


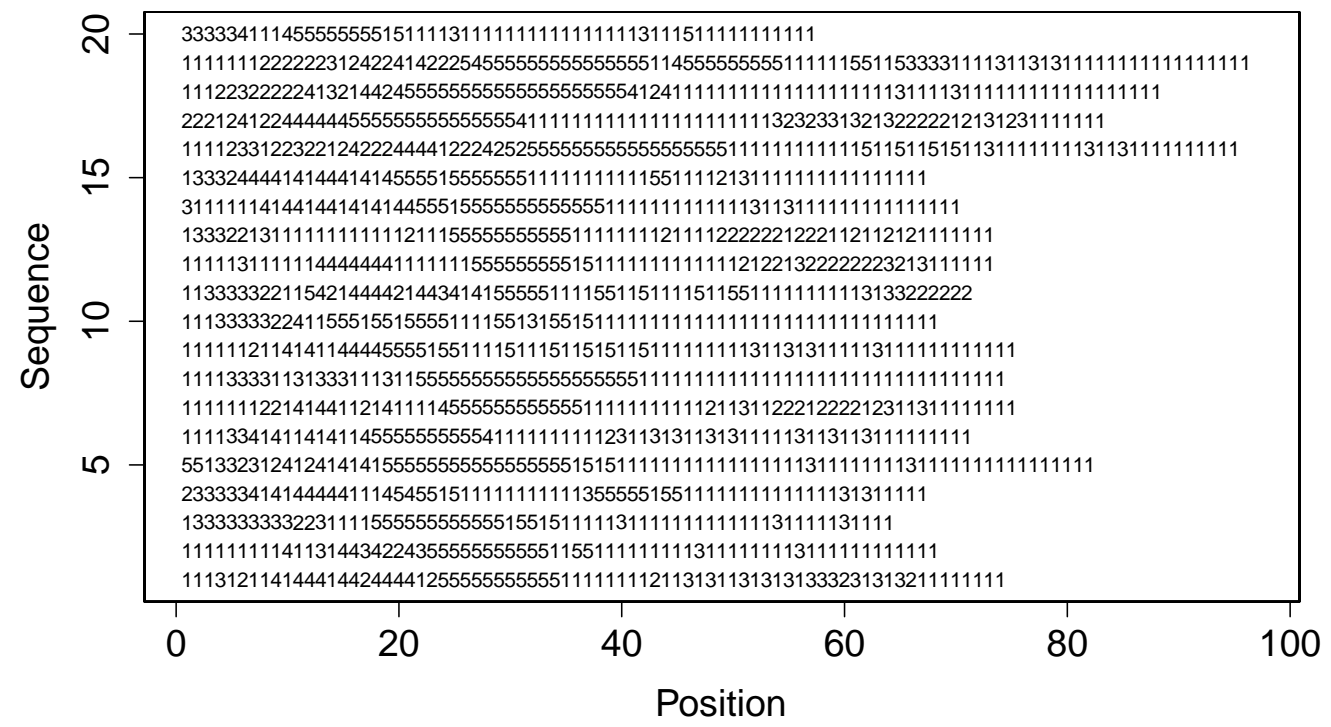

Figure 4: 20 apple tree sequences. For this figure sequences 1 to 18 were randomly chosen from the data set, while sequences 19 and 20 are the longest and the shortest sequences, respectively. As stated by Guédon et al. (2001) "the sequences may be viewed as a succession of homogeneous zones or segments where the composition properties do not change substantially within each zone, but change markedly between zones."

We analyzed 94 data sequences related to the branching of the main trunk of two-year old apple trees that are left without pruning for one year (Guédon 2003), and 20 of these sequences are shown in Figure 4. The main trunk consists of nodes (metamers or growth units), and each of these nodes can be categorized into one of five types of axillary production (or states): (1) latent bud, (2) 1-year-delayed short shoot, (3) 1-year-delayed-long shoot, (4) 1-year-delayed flowering shoot, and (5) immediate shoot. These different states correspond to nodes without any activity (state 1) and four different types of branching (states $2-5$ ). The data was recorded by examining the main trunk node by node from the top to the base (Godin et al. 1999). The lengths of the resulting sequences range from 57 to 96 observations as shown in Figure 4 .

Looking at the data in Figure 4 there is clearly some inhomogeneity in the observed sequences. For example, there is a tendency to have more occurrences of state 5 (immediate shoots) before the middle of each sequence, and the sequences seems to be changing between different states more frequently at the beginning (top of the main trunk) compared to at the end 


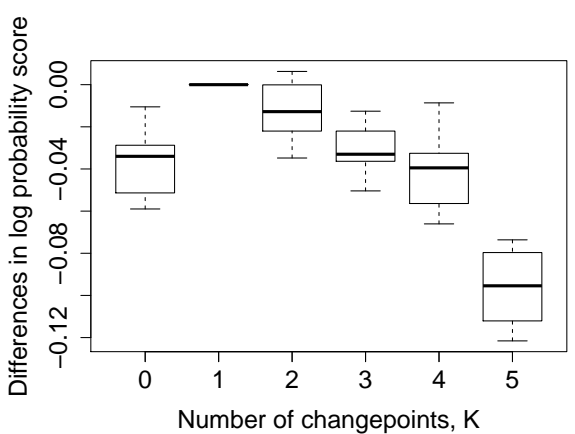

(a)

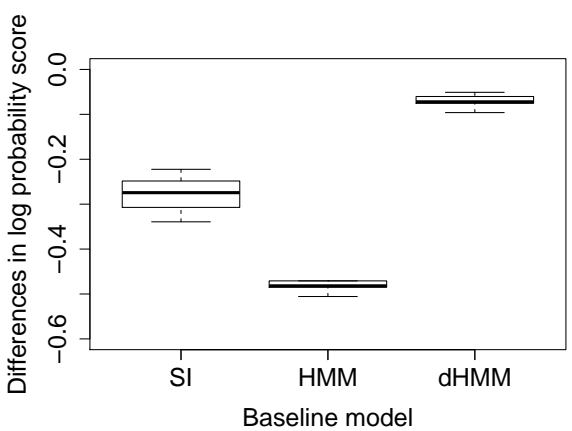

(b)

Figure 5: Each boxplot shows the log-probability scores, across the validation sets, for a different model. The $\mathrm{y}$-axis is defined as the log-probability score for (a) other numbers of changepoints and (b) other baseline models, minus the log-probability score for the model with one changepoint.

(base of the main trunk). However, it is not clear from visual inspection how many segments the data should be divided into, nor where changepoints between segments should occur. Using exploratory data analysis, Guédon et al. (2001) and Guédon (2003) suggested partitioning the sequences into six segments. As an alternative we can, in a data-driven manner, simultaneously determine the appropriate number of segments, the likely positions of these segments, and estimates of the Markov transition parameters within each segment.

To find the optimal number of changepoints for the model we randomly partition the sequences into ten test sets (and ten corresponding training sets). The split is done so that four test sets contain ten sequences and six test sets contain nine sequences. In Figure 5(a), we compare the cross-validated log probability scores of the model with one changepoint $(K=1)$ and models with zero, two, three, four, and five changepoints. We choose the $K=1$ model as the reference in this case because it was the model with the highest median log-probability score, i.e., the model with a single changepoint is the preferred model.

The cross-validated log-probability scores of the baseline models relative to the preferred model, are shown in Figure 5(b), Our model clearly outperforms the two baseline models using conditionally independent observations (SI and HMM) supporting the assumption that there 


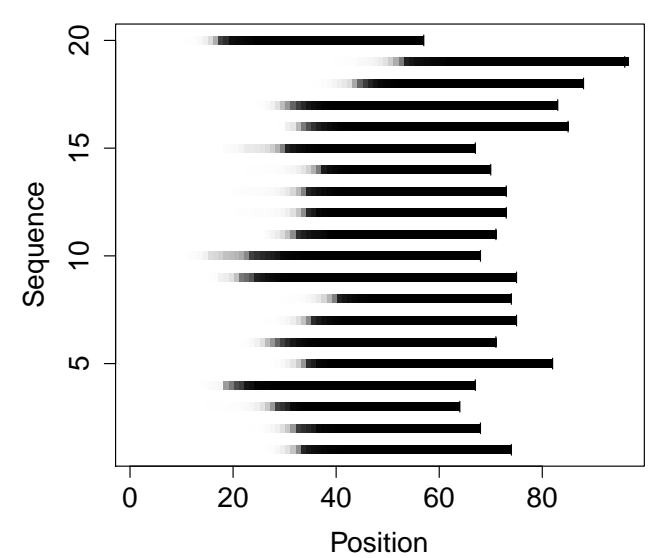

(a)

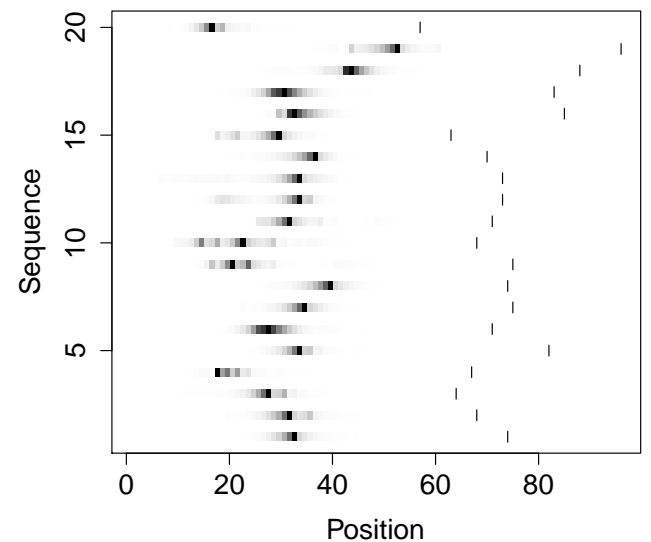

(b)

Figure 6: The estimated marginal probability distribution of (a) the classification of each node to segment 2 (probability 1 is black) and (b) a particular node being a changepoint, where the gray scale has been adjusted for each sequence so that the node with the maximum probability is black and the node with the minimum probability is white. The end position of each observed sequence is marked with a short vertical black line.

is Markov dependence in the observed sequences. Our model also performs better than the dHMM, suggesting that the negative binomial distribution for the changepoint locations is a better choice for this data set than the geometric distribution used by the dHMM and HMM. For a comparison between our joint model and the approach where each sequence is analyzed independently, see the supplementary materials Section S.2.2.

Using our proposed model with a single changepoint, we ran our sampling algorithm on all of the sequences in order to infer the position of the changepoint within each sequence and the transition matrix within each segment. Figure 6(a) shows the estimated marginal probability of each node being assigned to segment 2, for each of the 20 sequences from Figure 4. The estimated marginal distribution of the position of the changepoint within each of these sequences is shown in Figure 6(b). The results suggest that the changepoint tends to occur at a location roughly one quarter to one half of the length of the sequence from the start. None of the segments in any of the sequences where found to be skipped.

In Figure 7 we have superposed the observed data over the estimated marginal probability of each node being assigned to segment 2, for three selected sequences, namely the first sequence 

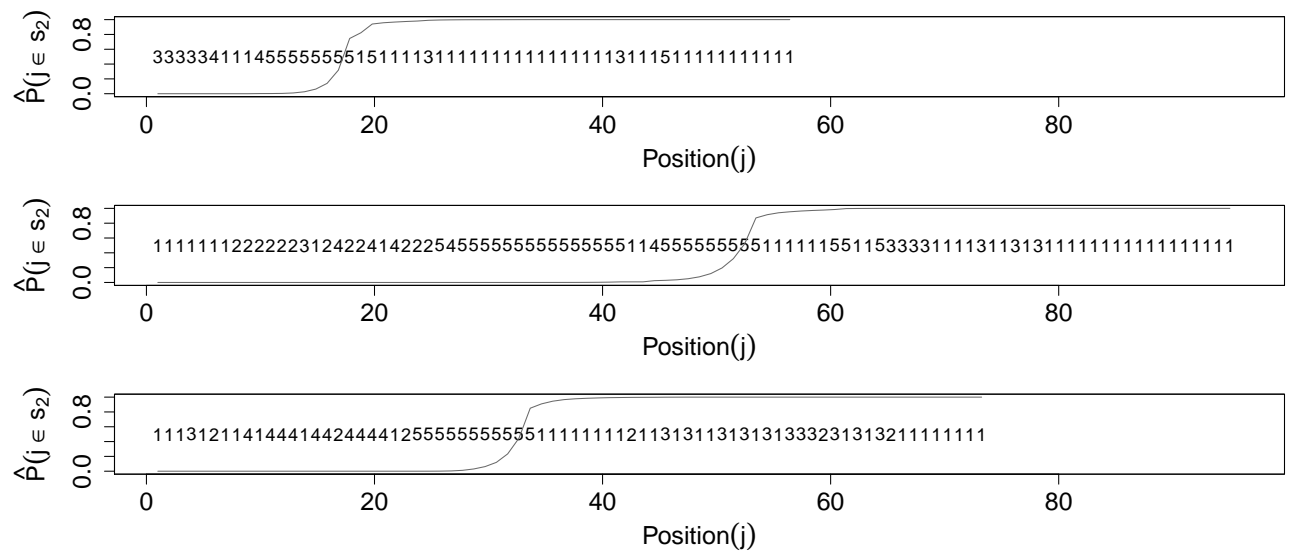

Figure 7: Sequence 1 (bottom), 19 (middle) and 20 (top) from Figure 4 with marginal estimates of the probability of each position being assigned to segment 2 (as a gray line).

in Figure 4, the longest sequence (sequence 19) in the same figure, and the shortest sequence (sequence 20). From the figure we can see that the changepoints are all placed after the phase where state 5 (immediate shoot) is frequently visited. Investigating the position of the six segments presented in Guédon et al. (2001) and Guédon (2003), our first and last segments correspond to the their first three and last three segments, respectively.

The estimates of the transition matrices for each segment,

$$
\hat{\boldsymbol{Q}}^{(1)}=\left[\begin{array}{ccccc}
0.54 & 0.07 & 0.10 & 0.20 & 0.09 \\
0.21 & 0.37 & 0.13 & 0.21 & 0.08 \\
0.17 & 0.10 & 0.50 & 0.17 & 0.06 \\
0.30 & 0.08 & 0.08 & 0.46 & 0.08 \\
0.09 & 0.03 & 0.01 & 0.03 & 0.84
\end{array}\right], \hat{\boldsymbol{Q}}^{(2)}=\left[\begin{array}{ccccc}
0.83 & 0.04 & 0.07 & 0.01 & 0.05 \\
0.31 & 0.36 & 0.22 & 0.05 & 0.06 \\
0.53 & 0.15 & 0.24 & 0.02 & 0.06 \\
0.30 & 0.19 & 0.07 & 0.26 & 0.18 \\
0.51 & 0.04 & 0.04 & 0.03 & 0.38
\end{array}\right]
$$

are consistent with the biology of tree growth. In segment 1, for example, the self-transition and transitions into state 4 ("flowering") are much higher than in segment 2. Since segment 2 corresponds to the lower part of the trunk, this makes sense since flowering is much less likely to occur towards the bottom of the trunk (segment 2) relative to the top (segment 1). We can also observe that the marginal probability of state 1 is much higher in segment 2 than in segment 1 
(via its self-transition and in-transition probabilities). State 1 is the non-branching state, so it is biologically reasonable to expect less branching in the lower part of the trunk (segment 2). The estimate for the changepoint parameters $r_{1}$ and $b_{1}$ are 0.416 and 0.652 , respectively, with $95 \%$ credible interval $[0.389,0.442]$ and $[0.456,0.867]$, respectively.

\subsection{Real Data Analysis: Monsoon Rainfall}

To illustrate the applicability of the model to a real-world problem we analyze the Indian monsoon data described earlier in Section 1. The onset and withdrawal of the annual summer monsoon is of critical importance in India since it directly impacts agricultural production, water resource management, and hydroelectricity production (Lima and Lall 2009). There is no precise definition of the monsoon season, but there is a general understanding that the onset is the time of consistent and substantial increase in rainfall over a regional area and the withdrawal is the time that marks the return to a dry period (Fasullo and Webster 2003;

Joseph et al. 2006). In terms of understanding the climatological variability of the monsoon over time, a first step is to label the changepoints of the onset and withdrawal in the historic record (Joseph et al. 2006), enabling (for example) prediction of onset and withdrawal as a function of exogenous variables such as large-scale atmospheric quantities (Pai and Nair 2009). Our proposed model provides a framework to not only detect but also to quantify our uncertainty about the estimation of the onset and withdrawal dates and our results can be viewed as an alternative to other approaches that use non-Markov models for this purpose (e.g., Stern (1982); Lima and Lall (2009)).

Finite-state Markov models have long been used to characterize daily rainfall occurrence in climatological applications (Gabriel and Neumann 1962; Katz 1974). Rainfall occurrence is typically defined as any amount more than $0.2 \mathrm{~mm}$ or $0.01 \mathrm{in}$. A second cutoff can be used to 
distinguish light and heavy rainfall events (anything over $20 \mathrm{~mm}$ is considered a heavy rainfall event). In our analysis below the daily rainfall amount is assigned to one of three discrete categories: no/light rainfall with $0-0.2 \mathrm{~mm}\left(y_{j}=1\right)$, medium rainfall with $0.2-20 \mathrm{~mm}\left(y_{j}=2\right)$, or heavy rainfall with $>20 \mathrm{~mm}\left(y_{j}=3\right)($ Katz 1977).

Our data set consists of daily rainfall measurements over a 31 year period (1979-2010) from a weather station located at latitude 24.65 and longitude 77.32 in the monsoon region of India 2 . The data is plotted in Figure 1 with each year plotted as a sequence. Rainfall amounts were categorized into three states as described above and the 153 missing observations (1.35\% of the data) were imputed during inference as described in Section S.1 in the supplementary materials. We denote each day of the year as $j=1, \ldots, 365$ (leap days are removed). We assume that the first and last segments in each year have the same Markov transition matrix, reflecting the fact that the end of one sequence on December 31 is contiguous in time with the start of the next sequence on January 1 . This constraint rules out the possibility of a single changepoint in the model, and thus, the number of possible changepoints we can consider is $\mathrm{K}=0,2,3,4, \ldots$..

To determine the optimal number of changepoints using cross-validation we randomly partitioned our sequences (or years) into 10 training/test sets where nine of the test sets contain three sequences and one has four sequences. Figure $8(\mathrm{a})$ compares the cross-validated log probability scores of the model with two changepoints, to models with zero, three, and four changepoints. The models with two and three changepoints are very close in performance and outperform models with zero or four changepoints. We select the model with two changepoints $(K=2)$ as our preferred model given that it is simpler and it corresponds to our physical intuition about the monsoon phenomenon (i.e. onset and withdrawal of the monsoon season).

\footnotetext{
${ }^{2}$ The data were obtained from the U.S. National Centers for Environmental Prediction (NCEP) Climate Prediction Center (CPC) Global Summary of the Day (GSOD) Observations, http://rda.ucar.edu/datasets/ds512.0
} 


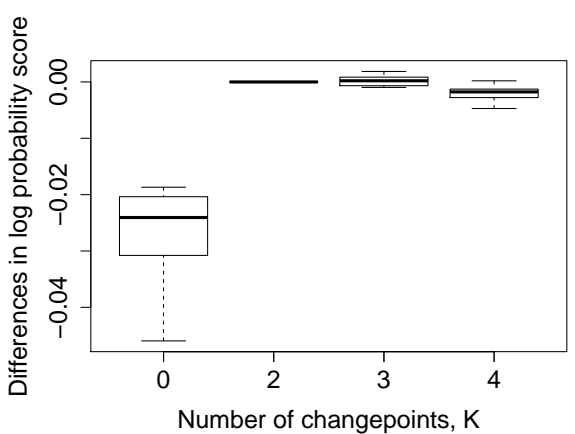

(a)

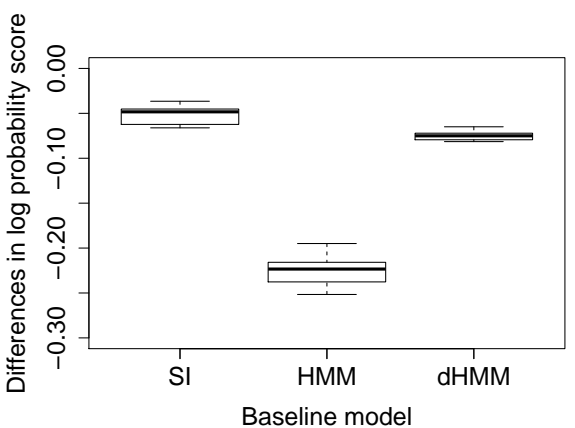

(b)

Figure 8: Each boxplot shows the log-probability scores, across the validation sets, for a different model. The $\mathrm{y}$-axis is defined as the log-probability score for (a) other numbers of changepoints and (b) other baseline models, minus the log-probability score for the model with two changepoints.

Figure 8(b) compares the model with two changepoints with the baseline models SI, HMM, and dHMM. As we can see, our proposed model significantly outperforms the three baselines. A comparison of the joint model and the approach based on individual sequences is provided in Section S.2.3 in the supplementary materials.

Figure 9(a) shows the estimates of the marginal probability of each day belonging to the monsoon season, providing a visual interpretation of the estimated interannual variability in the dates of the Indian monsoon onset and withdrawal across 31 years. The marginal distribution across all 31 years is shown at the top of the figure with each of the years plotted per row below. Most days in July and August, across all years, are highly probable to be classified as monsoon days (black) and the days at the beginning and end of the monsoon season have less certainty of being in the monsoon (gray).

The probability of each individual day being a changepoint (onset or withdrawal of the monsoon) can also be obtained from the model. Figure 9(b) shows the probability of a particular day being a changepoint in each of the 31 years. Days that are darker gray are more likely to be the monsoon onset or withdrawal date. The top line shows the marginal over all years. We see that the highest probability of onset is in June and the highest probability of withdrawal is 


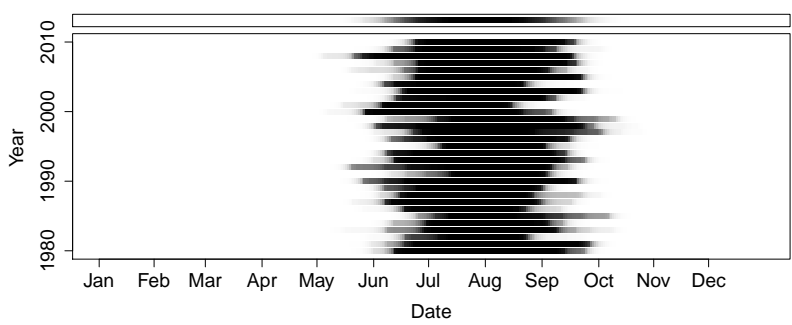

(a)

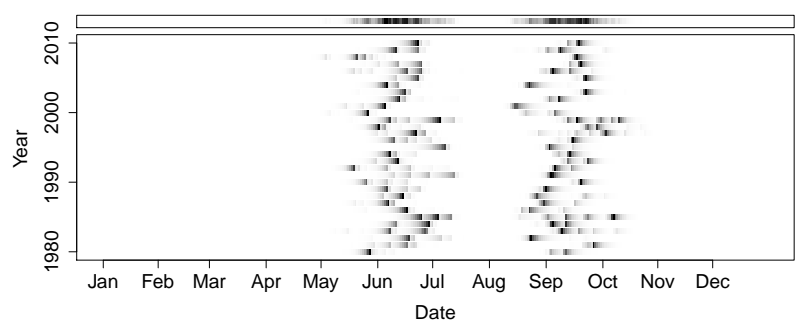

(b)

Figure 9: The estimated marginal probability distribution for (a) the classification of each day to the monsoon season (probability 1 is black) and (b) a particular day being a changepoint, where the gray scale has been adjusted for each year so that the day with the maximum probability is black and the day with the minimum probability is white.

in September.

The estimated transition matrices for our model with two changepoints, the first for the dry (non-monsoon) season and the second for the monsoon season are as follows

$$
\hat{\boldsymbol{Q}}^{d r y}=\left[\begin{array}{ccc}
0.916 & 0.026 & 0.002 \\
0.742 & 0.232 & 0.027 \\
0.423 & 0.367 & 0.210
\end{array}\right], \hat{\boldsymbol{Q}}^{\text {monsoon }}=\left[\begin{array}{ccc}
0.720 & 0.216 & 0.069 \\
0.439 & 0.436 & 0.125 \\
0.258 & 0.489 & 0.253
\end{array}\right]
$$

with rows/columns $1,2,3$, corresponding to no rainfall, light rainfall, and heavy rainfall categories respectively. The monsoon and non-monsoon seasons are distinctly different in terms of their Markov transition probabilities, with transitions into light and heavy rainfall categories being significantly higher in the monsoon season compared to the dry season. The estimates for the changepoint parameters $r_{1}, b_{1}, r_{2}$ and $b_{2}$ are $0.446,0.810,0.703$ and 0.885 , respectively, with $95 \%$ credible intervals $[0.431,0.461],[0.550,0.985],[0.687,0.719]$ and $[0.693,0.993]$, respectively.

Upon examining the model with three changepoints $(K=3)$, we found it added an additional changepoint in the middle of the monsoon season. However the estimated transition matrices for these two segments did not differ significantly from one another.

Our results are broadly consistent with other findings. For example, Fasullo and Webster 
(2003) analyzed data from a station further south in India, estimating that the monsoon onset dates at this location range from May 19 to June 20 with the median being June 6, and the withdrawal dates range from August 12 to September 27 with the median being September 4. These dates are consistent with our estimates at a more northerly location, given that the monsoon approaches from the south in India, and thus, we would expect our calculated onset and withdrawal dates to be somewhat later than those of Fasullo and Webster (2003). We obtain a mean estimate of June 13 for the monsoon onset with $95 \%$ probability interval (PI) of [May 23, July 4] and the mean date for the monsoon withdrawal is September 13 with an estimated 95\% PI of [August 24, October 3]. These estimates are based on the data shown at the top of Figure $9(\mathrm{~b})$ where the $95 \% \mathrm{PI}$ is computed by summing up the estimated marginal probabilities symmetrically around the mean until a $95 \%$ interval is reached.

\section{DISCUSSION AND CONCLUSIONS}

We introduced a piecewise homogeneous Markov chain model where changepoint positions are modeled by a discrete distribution, in particular, a truncated version of the negative binomial distribution. The model is constructed to handle multiple sequences of variable length where each sequence moves between the underlying Markov chains in the same order. We show that the changepoints are well recovered on synthetic data, resulting in accurate estimates for the parameters used to define our model. To illustrate the utility of the model on real-world data sets we applied the model to an apple tree branching data set and to daily rainfall data collected for 31 years in Northern India. In the apple tree data the model suggests that a single changepoint for each sequence is best, and for the rainfall data the model is able to detect the onset and withdrawal of the monsoon season. In both cases the proposed model produced inferences of parameters and changepoint locations that were scientifically interpretable. In 


\begin{tabular}{c|cccc} 
Data set & $K=0$ & $K=1$ & $K=2$ & $K=3$ \\
\hline Simulated Data 1 & 0.002 & 0.015 & 0.065 & 0.138 \\
Monsoon Data & 0.054 & 0.163 & 0.432 & 0.795
\end{tabular}

Table 1: Computational time per iteration in seconds for two of the data sets discussed in the paper, with code in Matlab on a computer with $2.80 \mathrm{GHz}$ CPU.

addition the model systematically outperformed alternative approaches such as hidden Markov and double-hidden Markov models.

Our Bayesian framework allows different sequences to draw strength from each other both when finding the changepoints and for parameter estimation. Estimates of uncertainty about both parameters and latent variables, which arise naturally from our MCMC inference algorithm, provide an appealing interpretation of the uncertainty regarding position of the changepoints. This is of particular interest for example in analyzing the onset and withdrawal of the Indian monsoon season. Uncertainty quantification is an increasingly important component of climate data analysis, and the type of Bayesian approach used in this paper can provide a useful data-driven alternative to more traditional methods such as using threshold values (Lima and Lall 2009) or relying entirely on definitions based on prior knowledge (Fasullo and Webster 2003).

Comparisons of the computation times for two of the data examples in the paper are shown in Table 1, comparing the times for the smallest data set (the first simulated data set) and the largest data set (the monsoon data). The numbers shown are for one MCMC iteration when fitting the model to the full data set (we used parallelization for our cross-validation runs). We see that as the number of changepoints increase the method is more computationally expensive, but overall the method is relatively fast even on the larger data set. Additional speedups could be obtained (for example) by parallelizing the analysis across sequences, coding the algorithm in a more efficient language than Matlab, and so on.

There are a number of additional extensions to the model that may be potentially useful 
to explore. For example, a useful direction would be the development of conditional models $p(\boldsymbol{y} \mid \boldsymbol{x})$, where each sequence $\boldsymbol{y}=\left(y_{1}, \ldots, y_{T}\right)$ is accompanied by a sequence $\boldsymbol{x}=\left(\boldsymbol{x}_{1}, \ldots, \boldsymbol{x}_{T}\right)$ of exogenous variables and where each $\boldsymbol{x}_{t}$ could be multivariate, $t=1 \ldots, T$. The exogenous variables $\boldsymbol{x}$ could influence the $\boldsymbol{y}$ 's directly and/or the locations of the changepoints. Another direction would be models for handling high-dimensional and/or real-valued observation sequences, where one could assume the existence of a categorical latent sequence $\boldsymbol{z}=\left(z_{1}, \ldots, z_{T}\right)$ as a low-dimensional representation of the observed sequence data $\boldsymbol{y}$, extending the general approach presented here to piecewise homogeneous hidden Markov chains.

\section{ACKNOWLEDGMENTS}

This work was supported in part by US Department of Energy award DOE-SC0006619 (TH and PS), US Office of Naval Research under MURI grant N00014-08-1-1015 (TH and PS), and US National Science Foundation award IIS-1320527 and a Google Faculty Award (PS).

\section{SUPPLEMENTARY MATERIALS}

Additional Supporting Information: Supplementary materials containing additional results, simulations, sensitivity analysis, etc. is given at the end of this document.

\section{REFERENCES}

Berchtold, A. (1999). "The double Markov chain." Communications in Statistics - Theory and Methods, 28, 2569-2589.

— (2002). "High-order extensions of the double chain Markov model." Stochastic Models, 18, $193-227$. 
Chen, J., Brissette, F., and Leconte, R. (2010). "A daily stochastic weather generator for preserving low-frequency of climate variability." Journal of Hydrology, 388, 480-490.

Cleynen, A., Koskas, M., Lebarbier, E., Rigaill, G., and Robin, S. (2014). "Segmentor3IsBack: an R package for the fast and exact segmentation of Seq-data." Algorithms for Molecular Biology, 9 .

Cleynen, A. and Lebarbier, E. (2013). "Segmentation of the Poisson and negative binomial rate models: a penalized estimator." arXiv preprint arXiv:1301.2534.

Cochran, W. (1938). "An extension of Gold's method of examining the apparent persistence of one type of weather." Quarterly Journal of the Royal Meteorological Society, 64, 631-634.

Craig, W. (1943). "The song of the Wood Pewee." Ph.D. thesis, Albany: University of the State of New York.

Durbin, R., Eddy, S., Krogh, A., and Mitchison, G. (1998). Biological Sequence Analysis: Probabilistic Models of Proteins and Nucleic Acids. Cambridge University Press.

Fasullo, J. and Webster, P. J. (2003). "A hydrological definition of Indian monsoon onset and withdrawal." Journal of Climate, 16, 3200-3211.

Fearnhead, P. (2006). "Exact and efficient inference for multiple changepoint problems." Statistics and Computing, 16, 203-213.

Fearnhead, P. and Liu, Z. (2007). "Online inference for multiple changepoint problems." Journal of the Royal Statistical Society, Series B, 69, 589-605.

- (2011). "Efficient Bayesian analysis of multiple changepoint models with dependence across segments." Statistics and Computing, 21, 217-229. 
Fitzpatrick, M. and Marchev, D. (2013). "Efficient Bayesian estimation of the multivariate double chain Markov model." Statistics and Computing, 23, 467-480.

Friel, N. and Rue, H. (2007). "Recursive computing and simulation-free inference for general factorizable models." Biometrika, 94, 661-672.

Gabriel, K. and Neumann, J. (1962). "A Markov chain model for daily rainfall occurrence at Tel Aviv." Quartly Journal of the Royal Meteorological Society, 88, 90-95.

Gelman, A., Hwang, J., and Vehtari, A. (2014). "Understanding predictive information criteria for Bayesian models." Statistics and Computing, 24, 997-1016.

Gneiting, T. and Raftery, A. E. (2007). "Strictly proper scoring rules, prediction, and estimation." Journal of the American Statistical Association, 102, 359-378.

Godin, C., Guédon, Y., and Costes, E. (1999). "Exploration of plant architecture databases with the AMAPmod software illustrated on an apple-tree hybrid family." Agronomie, 19, $163-184$.

Godin, C., Guédon, Y., Costes, E., and Caraglio, Y. (1997). "Measuring and analyzing plants with the AMAPmod software." In Plants to ecosystems - Advances in Computational Life Sciences, 2nd International Symposium on Computer Challenges in Life Science, ed. M. Michalewicz, 53-84. Melbourne, Australia: CSIRO Australia.

Gold, E. (1929). "Note on the frequency of occurrence of sequences in a series of events of two types." Quarterly Journal of the Royal Meteorological Society, 55, 307-309.

Green, P. J. (1995). "Reversible jump Markov chain Monte Carlo computation and Bayesian model determination." Biometrika, 82, 711-732. 
Guédon, Y. (2003). "Estimating hidden semi-Markov chains from discrete sequences." Journal of Computational and Graphical Statistics, 12, 604-639.

Guédon, Y., Barthelemy, D., Caraglio, Y., and Costes, E. (2001). "Pattern analysis in branching and axillary flowering sequences." Journal of Theoretical Biology, 212, 481-520.

Guttorp, P. (1995). Stochastic Modeling of Scientific Data. CRC Press.

Joseph, P. V., Eischeid, J. K., and Pyle, R. J. (1994). "Interannual variability of the onset of the Indian summer monsoon and its association with atmospheric features, El Niño, and sea surface temperature anomalies." Journal of Climate, 7, 81-105.

Joseph, P. V., Sooraj, K. P., and Rajan, C. K. (2006). "The summer monsoon onset process over South Asia and an objective method for the date of monsoon onset over Kerala." International Journal of Climatology, 26, 1871-1893.

Katz, R. W. (1974). "Computing probabilities associated with the Markov chain model for precipitation." Journal of Applied Meteorology, 13, 953-954.

— (1977). "Precipitation as a chain-dependent process." Journal of Applied Meteorology, 16, $671-676$.

Lai, T. L. and Xing, H. (2011). "A simple Bayesian approach to multiple change-points." Statistica Sinica, 21, 539-569.

Levin, E. and Pieraccini, R. (2000). "A stochastic model of human-machine interaction for learning dialog strategies." In IEEE Transactions on Speech and Audio Processing, 11-23.

Li, L., Qiu, S., Zhang, B., and Feng, C. X. (2014). "Approximating cross-validatory predictive evaluation in Bayesian latent variables models with integrated IS and WAIC." arXiv preprint $\operatorname{arXiv:1404.2918v4.}$ 
Lima, C. H. R. and Lall, U. (2009). "Hierarchical Bayesian modeling of multisite daily rainfall occurrence: rainy season onset, peak, and end." Water Resources Research, 45, W07422.

Luong, T.-M., Rozenholc, Y., and Nuel, G. (2013). "Fast estimation of posterior probabilities in change-point models through a constrained hidden Markov model." Computational Statistics and Data Analysis, 68, 129-140.

Neal, R. M. (2000). "Markov chain sampling methods for Dirichlet process mixture models." Journal of Computational and Graphical Statistics, 9, 249-265.

Newnham, E. (1916). "The persistence of wet and dry weather." Quarterly Journal of the Royal Meteorological Society, 42, 153-162.

Pai, D. S. and Nair, R. M. (2009). "Summer monsoon onset over Kerala: New definition and prediction." Journal of Earth System Science, 118, 123-135.

Polansky, A. M. (2007). "Detecting change-points in Markov chains." Computational Statistics and Data Analysis, 51, 6013-6026.

Rabiner, L. (1989). "A tutorial on hidden Markov models and selected applications in speech recognition." Proceedings of the IEEE, 77, 257-285.

Raftery, A. and Tavaré, S. (1994). "Estimation and modelling repeated patterns in high order Markov chains with the mixture transition distribution model." Applied Statistics, 43, 179199.

Rigaill, G., Lebarbier, E., and Robin, S. (2012). "Exact posterior distributions and model selection criteria for multiple change-point detection problems." Statistics and Computing, 22, 917-929. 
Stern, R. D. (1982). "Computing a probability distribution for the start of the rains from a Markov chain model for precipitation." Journal of Applied Meteorology, 21, 420-423.

Vehtari, A. and Lampinen, J. (2002). "Bayesian model assessment and comparison using crossvalidation predictive densities." Neural Computation, 14, 2439-2468.

Wilks, D. and Wilby, R. (1999). "The weather generation game: A review of stochastic weather models." Progress in Physical Geography, 23, 329-357.

Xing, H., Sun, N., and Chen, Y. (2012). "Credit rating dynamics in the presence of unknown structural breaks." Journal of Banking \& Finance, 36, 78-89.

Zhang, N. R. and Siegmund, D. O. (2007). "A modified Bayes information criterion with applications to the analysis of comparative genomic hybridization data." Biometrics, 63, $22-32$.

— (2012). "Model Sslection for high-dimensional, multi-sequence change-point problems." Statistica Sinica, 22, 1507-1538. 


\title{
Supplementary materials to the paper: Bayesian Detection of Changepoints in Finite-State Markov Chains for Multiple Sequences
}

\author{
Petter Arnesen, Tracy Holsclaw and Padhraic Smyth 『
}

\section{S.1. SAMPLING ALGORITHM FOR POSTERIOR INFERENCE}

For notational convenience we assume in this section that the number of observed sequences is $L=1$. The generalization to sampling with multiple sequences is straightforward given the assumption of conditionally independent sequences from Section 2.4. We use the MetropolisHasting algorithm in all of our sampling updates.

The changepoints $\tau_{i}$ are sampled by proposing a new position $\tilde{\tau}_{i}=\tau_{i}+1$ or $\tilde{\tau}_{i}=\tau_{i}-1$, both with probability 0.5 , with obvious adjustments if there are zero-length segments involved in the update. Letting $\boldsymbol{\tau}=\left(\tau_{1}, \ldots, \tau_{i}, \ldots, \tau_{K}\right)$ be the current segmentation and $\tilde{\boldsymbol{\tau}}=\left(\tau_{1}, \ldots, \tilde{\tau}_{i}, \ldots, \tau_{K}\right)$ be the proposed new segmentation, this yields the following acceptance probability

$$
\operatorname{acc}(\tilde{\boldsymbol{\tau}} \mid \boldsymbol{\tau})=\min \left\{1, \frac{p\left(\boldsymbol{y} \mid \tilde{\boldsymbol{\tau}}, \overline{\mathbf{Q}}, y_{0}\right) p(\tilde{\boldsymbol{\tau}} \mid T, \overline{\boldsymbol{\theta}}) q(\boldsymbol{\tau} \mid \tilde{\boldsymbol{\tau}})}{p\left(\boldsymbol{y} \mid \boldsymbol{\tau}, \overline{\mathbf{Q}}, y_{0}\right) p(\boldsymbol{\tau} \mid T, \overline{\boldsymbol{\theta}}) q(\tilde{\boldsymbol{\tau} \mid \boldsymbol{\tau})}}\right\},
$$

where $q(\tilde{\boldsymbol{\tau}} \mid \boldsymbol{\tau})$ is the probability of proposing $\tilde{\boldsymbol{\tau}}$ given that the current state is $\boldsymbol{\tau}$.

To update $r_{i}$ we adopt the following strategy. First we propose a new value $\tilde{r}_{i}$ from a proposal distribution of the form $\left(\tilde{r}_{i}, 1-\tilde{r}_{i}\right) \mid r_{i} \sim \operatorname{Dir}\left(a_{r}\left(r_{i}, 1-r_{i}\right)\right)$, where $a_{r}$ is a tuning parameter that

Petter Arnesen is a PhD student with the Department of Mathematical Sciences, Norwegian University of Science and Technology, Trondheim 7491, Norway (email: petterar@math.ntnu.no). Tracy Holsclaw is a Postdoctoral Scholar with the Department of Statistics, University of California, Irvine, CA (email: tholscla@ams.ucsc.edu). Padhraic Smyth is a Professor with the Department of Computer Science and the Department of Statistics, University of California, Irvine, CA (email: smyth@ics.uci.edu). 
controls how close the proposed value $\tilde{r}_{i}$ will be to the current value $r_{i}$. The same strategy is used when proposing new values for $b_{i}$, using a separate tuning parameter $a_{b}$. To update the values in the transition matrix, $\boldsymbol{Q}^{(i)}$, we propose to change one of the $n$ rows. For example, $\boldsymbol{Q}_{k, \text {, }}^{(i)}$ is updated by a proposal distribution of the form $\tilde{\boldsymbol{Q}}_{k, .}^{(i)} \mid \boldsymbol{Q}_{k, \text {, }}^{(i)} \sim \operatorname{Dir}\left(a_{Q} \boldsymbol{Q}_{k,}^{(i)}\right)$, where $a_{Q}$ is a tuning parameter controlling the acceptance rates for such updates. For the results reported in this paper we set tuning parameter values to $a_{r}=1000, a_{b}=100$, and $a_{Q}=1000$. These values were chosen to obtain fast convergence and good mixing of the MCMC chain, and we found that the algorithm is not particularly sensitive to these values. In particular, a five-factor change in these values does not significantly effect the sampling.

We define one iteration of our algorithm to be a single proposed update of $b_{i}$ and $r_{i}$ for one value of $i=1, \ldots, K$, a single proposed update for one of the rows in one of the transition matrices, and $V$ proposed updates of each of the changepoints in each sequence. We found empirically that using $V>1$ updates leads to faster convergence and better mixing of the MCMC sampler (we used $V=5$ for the results in this paper). Results are obtained from 100,000 iterations of the MCMC chain after removing a burn-in period of 10,000 iterations. In addition, we do thinning by using only every 100th iteration in order to obtain independent samples.

In practice it is common to have missing data (for example for the rainfall data discussed in the paper) motivating the use of a systematic approach for handling such missing observations. We marginalize over missing observations in our sampling algorithm whenever we need to evaluate the data likelihood in (3). The calculation is carried out using a version of the recursive algorithm given in Friel and Rue (2007). Let $m=\left\{j \mid y_{j}\right.$ is missing $\}$, and let $y_{j}^{*}$ denote the actual value of a non-missing variable $y_{j}$. We write $p\left(y_{j}=y_{j}^{*} \mid y_{j-1}, \boldsymbol{\tau}, \overline{\mathbf{Q}}\right)$ when evaluating the probability of that event. When marginalizing over missing values the data likelihood of the 
non-missing values $\boldsymbol{y}_{-m}$ becomes

$$
p\left(\boldsymbol{y}_{-m} \mid \boldsymbol{\tau}, \overline{\mathbf{Q}}, y_{0}\right)=\sum_{y_{j}: j \in m} p\left(\boldsymbol{y} \mid \boldsymbol{\tau}, \overline{\mathbf{Q}}, y_{0}\right) .
$$

To evaluate this let $z_{0}\left(y_{1}\right)=p\left(y_{1} \mid y_{0}\right)$, and for $j=1, \ldots, T-1$ we define recursively

$$
z_{j}\left(y_{j+1}\right)= \begin{cases}p\left(y_{j+1} \mid y_{j}=y_{j}^{*}\right) z_{j-1}\left(y_{j}=y_{j}^{*}\right) & y_{j} \text { is not missing } \\ \sum_{y_{j}} p\left(y_{j+1} \mid y_{j}\right) z_{j-1}\left(y_{j}\right) & y_{j} \text { is missing. }\end{cases}
$$

To complete the calculation we find

$$
p\left(\boldsymbol{y}_{-m}=\boldsymbol{y}_{-m}^{*} \mid \overline{\mathbf{Q}}, \boldsymbol{\tau}, y_{0}\right)= \begin{cases}z_{T-1}\left(y_{T}=y_{T}^{*}\right) & y_{T} \text { is not missing } \\ \sum_{y_{T}} z_{T-1}\left(y_{T}\right) & y_{T} \text { is missing. }\end{cases}
$$

\section{S.2. ADDITIONAL POSTERIOR RESULTS FOR THE EXAMPLES IN THE PAPER}

\section{S.2.1 Synthetic Data: Scenario 1}

Parameters $b$ and $r$ are correlated with one another thus making their values interdependent. Because of this interdependence we investigate below the marginals and the sensitivity of the model relative to these parameters. Both parameters are well estimated, although there is considerable uncertainty concerning the estimated value of $b$ (see Table S.1). Figure S.1(a) shows, however, that the distribution of the changepoints, $p(\boldsymbol{\tau} \mid T=200, r=0.5, b)$, is not particularly sensitive to the value of $b$ within the credibility interval. The parameter $r$ primarily controls the position of the changepoints. Sensitivity analysis for this parameter is shown in 


\begin{tabular}{c|ccc} 
& True & Est. & \multicolumn{1}{c}{$95 \%$ CI } \\
\hline$r_{1}$ & 0.5 & 0.458 & $0.406,0.513$ \\
$b_{1}$ & 0.8 & 0.615 & $0.444,0.927$
\end{tabular}

Table S.1: Parameter estimate and 95\% CI.

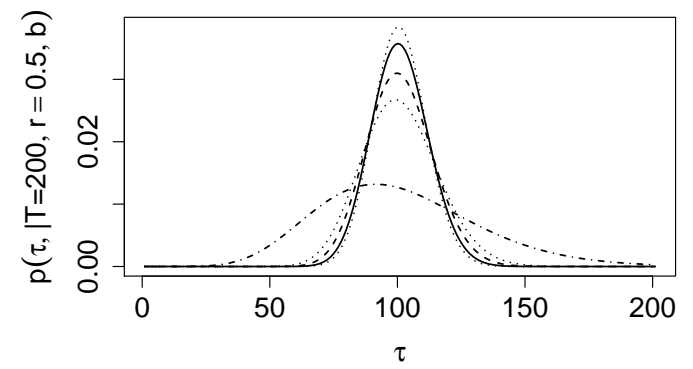

(a)

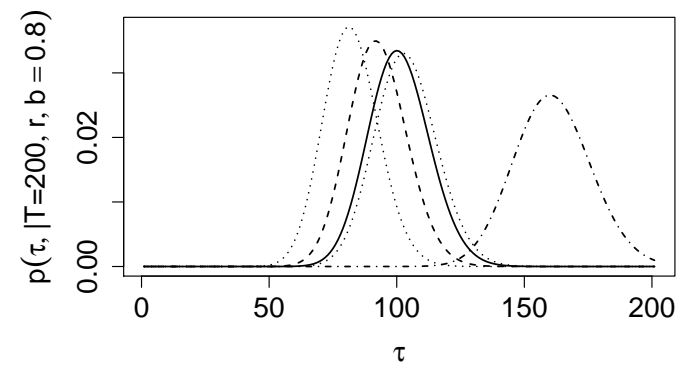

(b)

Figure S.1: (a) Sensitivity of the probability distribution $p(\boldsymbol{\tau} \mid T=200, r=0.5, b)$. The solid line corresponds to $b=0.8$ (true value), dashed line to $b=0.615$ (estimated value), and the dotted lines correspond to $b=0.444$ and $b=0.927$ (limits of the 95\% CI). The dash-dotted line corresponds to $b=0.1$. (b) Sensitivity of the probability distribution $p(\boldsymbol{\tau} \mid T=200, r, b=0.8)$. The solid line corresponds to $r=0.5$ (true value), dashed line to $r=0.458$ (estimated value), and the dotted lines correspond to $r=0.406$ and $r=0.513$ (limits of the $95 \% \mathrm{CI}$ ). The dash-dotted line corresponds to $r=0.8$.

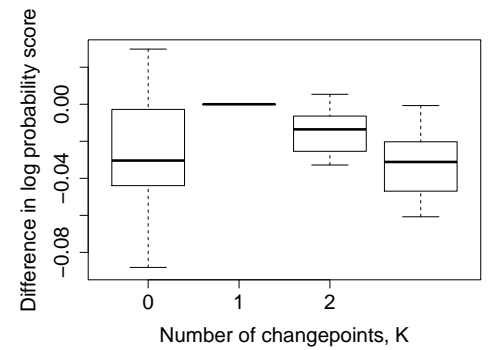

(a)

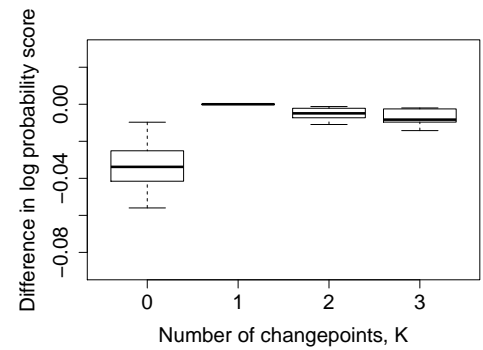

(b)

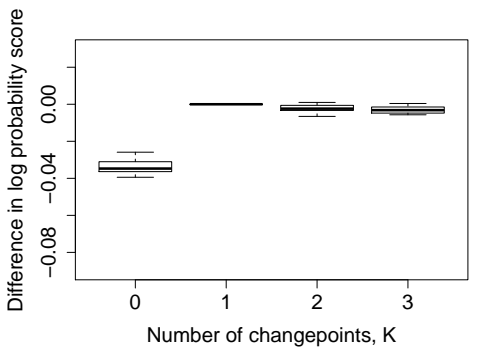

(c)

Figure S.2: Each boxplot shows the log-probability scores, across the validation sets, for a different model. The y-axis is defined as the log-probability score for other numbers of changepoints minus the log-probability score for the model with one changepoint, and the three plots correponds to different number of sequences $L$ : (a) $\mathrm{L}=5$, (b) $\mathrm{L}=20$ and (c) $\mathrm{L}=100$.

Figure S.1(b). The relatively high posterior uncertainty for these two parameters is somewhat due to the small sample size of only 10 sequences. We also generated a data set with $L=100$ sequences and found that the estimate for $b$ is 0.712 with a $95 \%$ CI of $(0.555,0.902)$, and as expected the extra sequences improve the accuracy and precision of the estimate.

We also varied $L$ in this scenario to test the sensitivity of the model to the number of sequences. We generated data sets with $L=5, L=20$, and $L=100$ and display the results in 
Figure S.2. For the data sets with $L=20$ and $L=100$ the results are similar to Figure 2(a) with $L=10$. For $L=5$ the cross-validation test tends to prefer the correct solution $(K=1)$ (where we generated 15 folds in total, comprised of validation sets with either a single sequence or a pair of sequences), although $K=0$ (no changepoint) is preferred in some cases, likely due to limited availability of data to support a more complex model.

In addition to comparing our model to the baseline models presented in the paper we also compare our results to those obtained with a model where each sequence is analyzed independently. In particular, we use the single-sequence model of Fearnhead (2006) for comparison, originally proposed for real-valued data with independence. We adapt this model by conditioning on the number of changepoints and extending to a Markov assumption. In the Fearnhead model, the prior assumes a geometric distribution to the length of each segment. By fixing the number of changepoints this simply becomes a uniform distribution on the position of the changepoints. Note that this is a special case of our model applied to single sequences when $b_{i}$ approaches zero for all $i$. Figure $\mathbf{S . 3}$ shows the results from running this model with a fixed (correct) number of changepoints, and the results can be compared to those of Figure 3 in the paper. Comparing these figures illustrates the strength of our joint modeling approach. Since our model is able to draw strength across sequences we achieve more accurate estimates of the position of the changepoints compared to the single sequence model. Similar results can be shown for the estimate of the transition matrices in each of the segments (see Table S.2 ). In this table we clearly see that the estimates for the transition probabilities for the individual sequences have a much higher uncertainty than the estimates for our joint model approach (shown in the last row). 


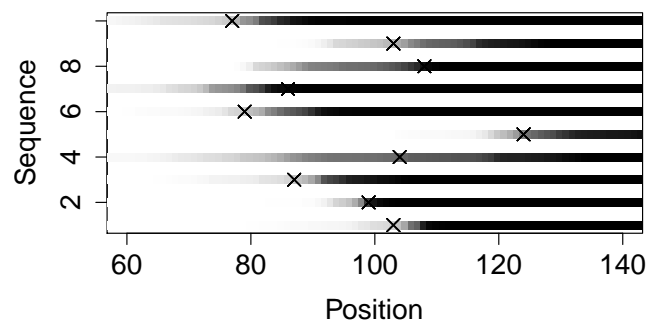

(a)

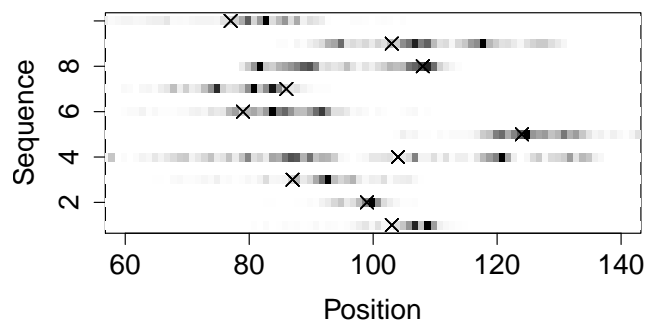

(b)

Figure S.3: The estimated marginal probabilities for individual sequence model of (a) the classification of each observation to segment 2 (probability 1 is black) and (b) a particular position being a changepoint, where the gray scale has been adjusted for each sequence so that the position with the maximum probability is black and the position with the minimum probability is white. The true changepoint locations are marked with $(\times)$ in both plots. Note that only position 60 to 140 are shown in each sequence.

\begin{tabular}{c|cccc} 
Seq. & $\hat{q}_{1,1}(95 \% \mathrm{CI})$ & $\hat{q}_{2,1}(95 \% \mathrm{CI})$ & $\hat{q}_{1,2}(95 \% \mathrm{CI})$ & $\hat{q}_{2,2}(95 \% \mathrm{CI})$ \\
\hline 1 & $0.856(0.757,0.907)$ & $0.711(0.580,0.824)$ & $0.462(0.351,0.582)$ & $0.190(0.113,0.300)$ \\
2 & $0.770(0.654,0.840)$ & $0.725(0.608,0.829)$ & $0.392(0.295,0.497)$ & $0.389(0.284,0.506)$ \\
3 & $0.842(0.751,0.910)$ & $0.760(0.628,0.876)$ & $0.504(0.393,0.614)$ & $0.430(0.323,0.530)$ \\
4 & $0.820(0.722,0.895)$ & $0.844(0.738,0.928)$ & $0.601(0.446,0.717)$ & $0.447(0.316,0.565)$ \\
5 & $0.764(0.682,0.849)$ & $0.739(0.645,0.816)$ & $0.506(0.389,0.646)$ & $0.484(0.392,0.620)$ \\
6 & $0.807(0.689,0.890)$ & $0.701(0.576,0.798)$ & $0.565(0.464,0.658)$ & $0.393(0.295,0.504)$ \\
7 & $0.866(0.777,0.928)$ & $0.713(0.557,0.840)$ & $0.475(0.366,0.606)$ & $0.371(0.276,0.483)$ \\
8 & $0.802(0.690,0.884)$ & $0.842(0.741,0.917)$ & $0.651(0.5440,0.746)$ & $0.326(0.216,0.472)$ \\
9 & $0.846(0.746,0.898)$ & $0.621(0.395,0.763)$ & $0.513(0.390,0.629)$ & $0.480(0.340,0.606)$ \\
10 & $0.811(0.668,0.929)$ & $0.885(0.789,0.947)$ & $0.512(0.395,0.627)$ & $0.379(0.271,0.475)$ \\
\hline Joint & $0.829(0.792,0.852)$ & $0.772(0.742,0.810)$ & $0.514(0.475,0.550)$ & $0.382(0.344,0.423)$
\end{tabular}

Table S.2: Parameter estimate and 95\% CI for the transition probabilities estimated based on individual sequences (Seq. 1-10), and for our joint model approach (Joint).

\section{S.2.2 Real Data Analysis: Branching of apple Trees}

In this section we present results from running the individual sequence approach on the apple tree data set. In Figure $\mathrm{S.4}$ we show the estimates for the position of the changepoints using a model with $K=1$, and this is to be compared with the result from our joint model shown in Figure 6 in the paper. From these figures we see that the individual sequence approach approximately finds the same changepoints as the joint model approach, although with somewhat higher uncertainty. Note that for some of the sequences, for instance sequences 5 and 12 in 


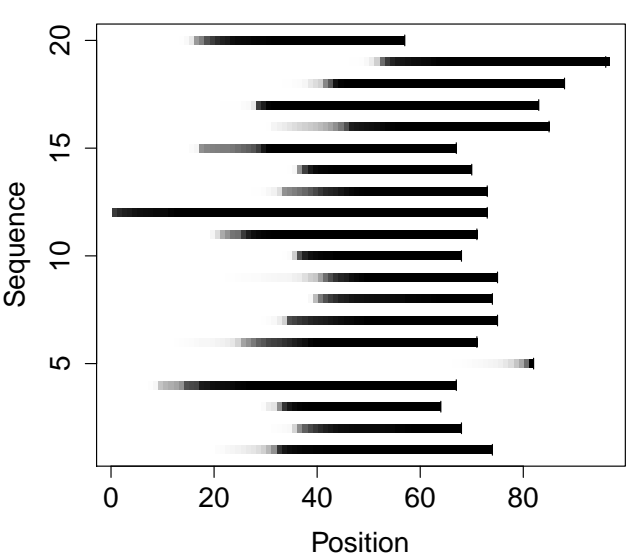

(a)

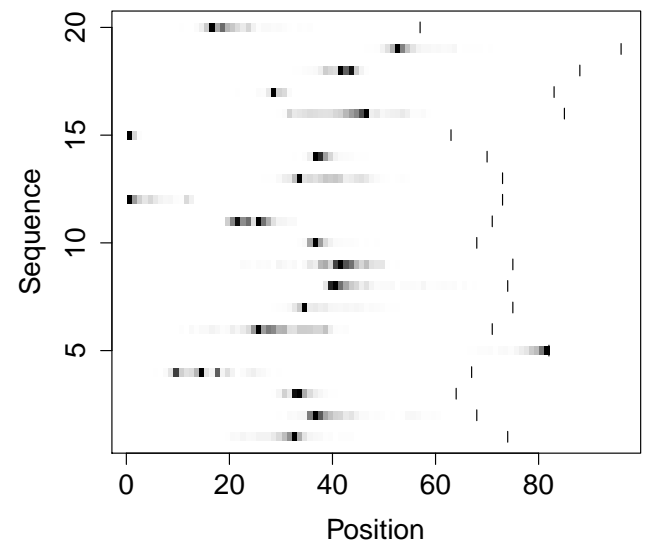

(b)

Figure S.4: The estimated marginal probability distribution of (a) the classification of each node to segment 2 (probability 1 is black) and (b) a particular node being a changepoint, where the gray scale has been adjusted for each sequence so that the node with the maximum probability is black and the node with the minimum probability is white. The end position of each observed sequence is marked with a short vertical black line.

Figure S.4 the individual sequence approach converges into a state with a zero length segment, which is somewhat surprising as there seems to be no significant difference in the structure of these two sequences compared to the others (see Figure 4 in the paper). This result is not present in our joint approach due to the sharing of information between the sequences. Also, because this is categorical data with five states the uncertainty in the estimates of the transition probabilities for the individual sequence approach becomes quite high due to lack of data, compared to our joint approach.

\section{S.2.3 Real Data Analysis: Monsoon Rainfall}

We also applied the individual sequence approach to the rainfall data presented in Section 4.3. in the paper. We assumed for this experiment that $K=2$ and the results are shown in Figure S.5. This figure clearly shows very high uncertainty regarding the position of the changepoints, and in most of these cases these changepoints are completely unrelated to the actual onset and offset of the monsoon season. This figure should be compared to the results using our joint approach shown in Figure 9 in the main paper. 


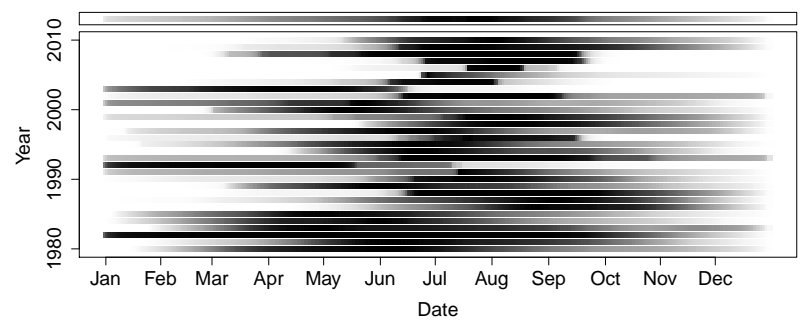

(a)

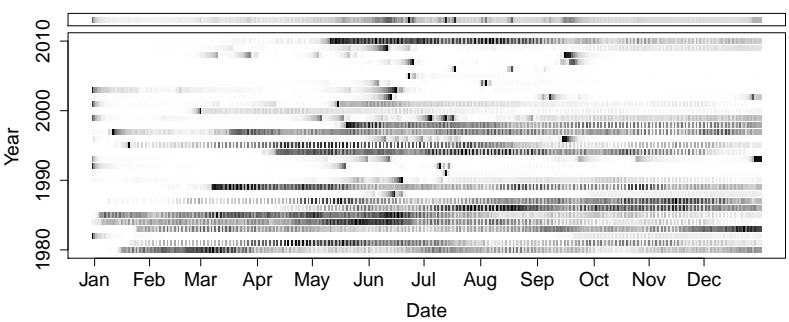

(b)

Figure S.5: The estimated marginal probability distribution for (a) the classification of each day to the monsoon season (probability 1 is black) and (b) a particular day being a changepoint, where the gray scale has been adjusted for each year so that the day with the maximum probability is black and the day with the minimum probability is white.

\section{S.3. ADDITIONAL SYNTHETIC AND REAL DATA ANALYSIS EXAMPLES}

\section{S.3.1 Synthetic Data Example: Scenario 2}

In this scenario, we generate binary data from our model for $L=30$ sequences with length $T_{l}=200$. The first 20 sequences are generated with two changepoints and the last 10 sequences are generated with only one changepoint. The 10 sequences with one changepoint are generated using the same parameters as described in Section 4.1 in the paper. The 20 sequences with two changepoints have similar first and last segments (as in Section 4.1) plus an additional middle segment where $\left(r_{2}, b_{2}\right)=(0.75,0.8)$ and $q_{1,2}=q_{2,2}=0.2$.

We used 10-fold cross validation to find the optimal number of changepoints. In this scenario $K=2$ effectively represents the true maximal number of changepoints in any sequence, where sequences with fewer changepoints can skip the "extra" segments. Thus, the interpretation of a single "overall best $K$ " has less meaning in this context. Nonetheless, using $K=2$ changepoints as the reference, Figure S.6(a) shows that two changepoints $(K=2)$ are preferred using the cross-validation score, which is consistent with the fact that 20 out of the 30 sequences were generated with two changepoints. Figure $\mathrm{S} 6(\mathrm{~b})$ shows that our model outperforms the other 


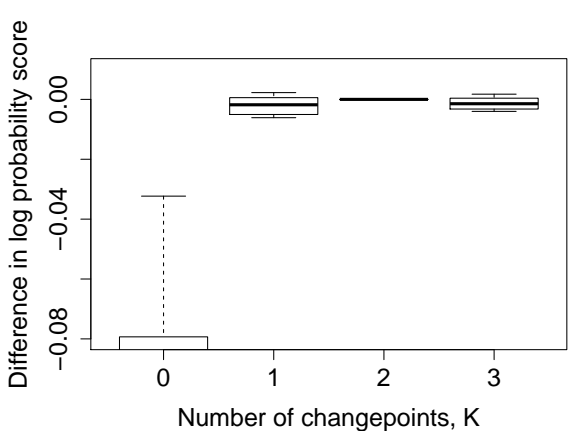

(a)

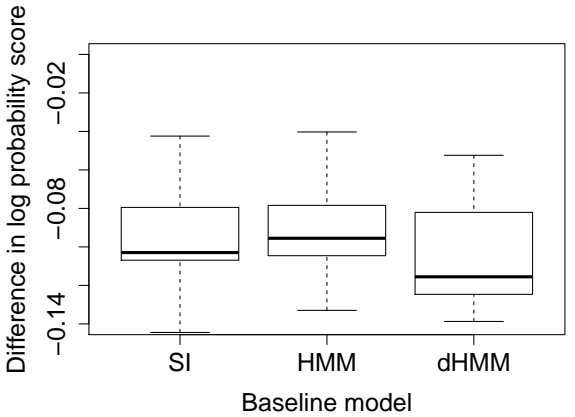

(b)

Figure S.6: Each boxplot shows the log-probability scores, across the validation sets, for a different model. The $\mathrm{y}$-axis is defined as the log-probability score for (a) other numbers of changepoints and (b) other baseline models, minus the log-probability score for the model with two changepoints.

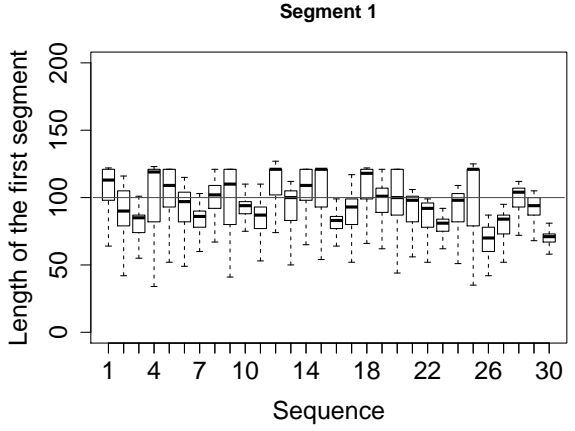

(a)

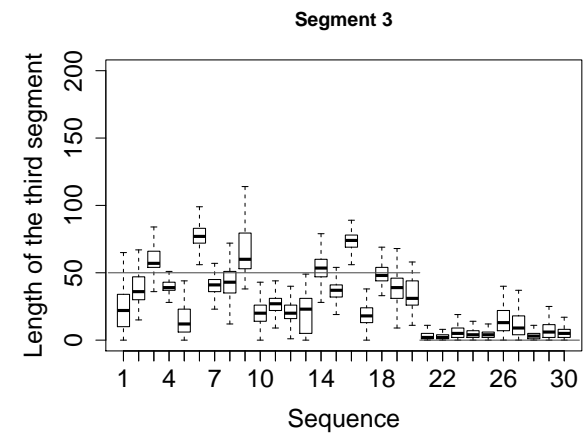

(c)

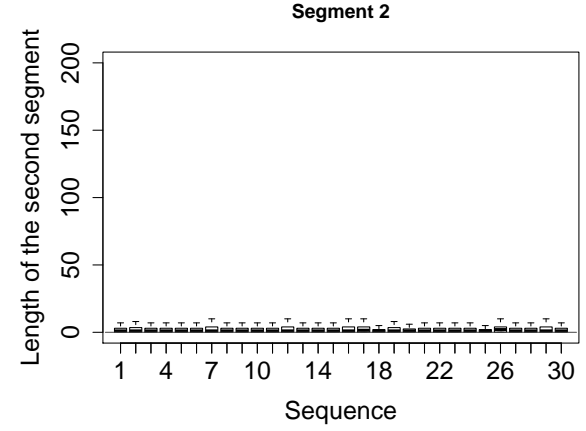

(b)

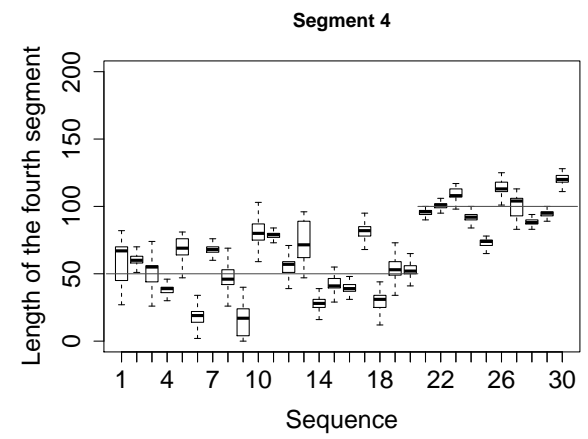

(d)

Figure S.7: Distribution of the length of each segments in each sequence in the $K=3$ solution. a)-d) are segments 1 to 4 respectively. Each sequence has a box plot for the posterior estimate of the length of its segment. For simplicity we show the expected length of each segment as a solid gray line, noting that for each simulated sequence the actual segment length will vary around this expected length.

baseline models, all fit with $K=2$.

To illustrate how our model skips segments, we examine in detail the solutions found by the model when it is fit to all 30 sequences and allowed to use $K=3$ changepoints, even though the 


\begin{tabular}{c|cc} 
& Est. & $95 \%$ CI \\
\hline$r_{1}$ & 0.382 & $0.225,0.512$ \\
$b_{1}$ & 0.618 & $0.242,0.832$ \\
$r_{2}$ & 0.412 & $0.245,0.538$ \\
$b_{2}$ & 0.858 & $0.510,0.901$ \\
$r_{3}$ & 0.732 & $0.556,0.904$ \\
$b_{3}$ & 0.004 & $0.001,0.012$ \\
$q_{1,1}$ & 0.779 & $0.768,0.804$ \\
$q_{2,1}$ & 0.803 & $0.786,0.821$ \\
$q_{1,2}$ & 0.722 & $0.365,0.846$ \\
$q_{2,2}$ & 0.708 & $0.322,0.834$ \\
$q_{1,3}$ & 0.216 & $0.194,0.239$ \\
$q_{2,3}$ & 0.184 & $0.156,0.209$ \\
$q_{1,4}$ & 0.496 & $0.443,0.546]$ \\
$q_{2,4}$ & 0.369 & $0.312,0.422]$
\end{tabular}

Table S.3: Parameter estimate and 95\% CI.

data was generated with only $K=1$ or $K=2$ changepoints. Figure $\mathbf{S . 7}$ shows the results of this experiment, with each of the four panes corresponding to one of the four possible segments that the model could infer. The estimated posterior distribution of the length of each segment is shown in the form of a box plot (the $y$-axis) for each of the 30 sequences (the $x$-axis). Recall that sequences 1 to 20 were generated with $K=2$ changepoints and sequences 21 to 30 with $K=1$ changepoint. The expected length of each segment given the true changepoint model is shown as a gray line. From the figure we see that the second segment (Figure $\underline{S .7}(\mathrm{~b})$ ) is skipped for all sequences, i.e., the model finds no evidence for an additional segment. Similarly, in Figure $\underline{S .7}(\mathrm{c})$ we see that sequences 21 to 30 infer very short or zero length segments for the third segment, and as a consequence the fourth segment is much longer in these sequences (Figure S.7(d)). Thus, even with misspecification of the value of $K$, and when sequences have variable numbers of changepoints, our model appears to perform well.

For the posterior distributions of the parameters, we see some summary statistics in Table S.3. For these statistics we find many similarities with the true parameters of the data. For instance, the $95 \%$ CIs of $r_{1}$ and $b_{1}$ include the true parameter of the first changepoint for both types of sequences. Next, $r_{2}$ and $b_{2}$ correspond to the changepoint leading up to the first (often) 
zero length segment, so it is reasonable that $r_{2}$ is close to $r_{1}$. Next, we see a high uncertainty in the position of the third changepoint, $b_{3}=0.004$, which makes sense because this is the changepoint leading up to the third changepoint, which is approximately of length 50 for the 20 first sequences and (often) zero for the last 10 sequences. For the transition matrix parameters we see high uncertainty for the estimates of $q_{1,2}$ and $q_{2,2}$ which makes sense because this segment is (often) zero for all the sequences. All other transition matrix parameters are well recovered.

We also investigated both the $K=2$ and the $K=1$ solution for this data set. For the $K=2$ case, one segment is skipped for the last 10 sequences, as expected. For the $K=1$ solution the position of the single changepoint in the last 10 sequences gets well estimated, while in the first 20 sequences the position of the first changepoint is the one that is recovered accurately and the model merges the second and third segments.

\section{S.3.2 Synthetic Data Example: Scenario 3}

In this section we present an experiment similar to what is done in the previous section (Section S.3.1), however we switch the number of sequences with $K=1$ changepoints with the number of sequences with $K=2$ changepoints. In Figure $\mathbf{S . 8}$ we see the result from our cross validation procedure for different numbers of changepoints, and with comparisons to the baseline models. We observe $K=2$ to be the superior choice. This is slightly surprising considering that the example in Section S.3.1 gave a less significant difference between the $K=1$ and $K=2$ solution even though in that case there were more sequences with $K=2$ changepoints. We believe that this is likely to result from differences between these two random data sets.

In Figure $\$$.9 we see the posterior estimation of the length of each segments when we assume a model with $K=3$ changepoints, again a similar procedure as in Section S.3.1. Again the zero length segments can be observed. 


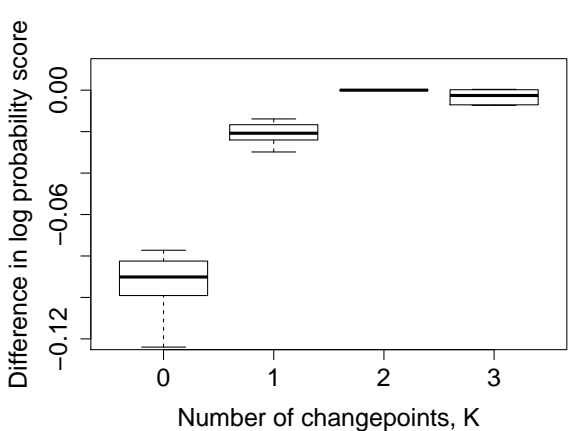

(a)

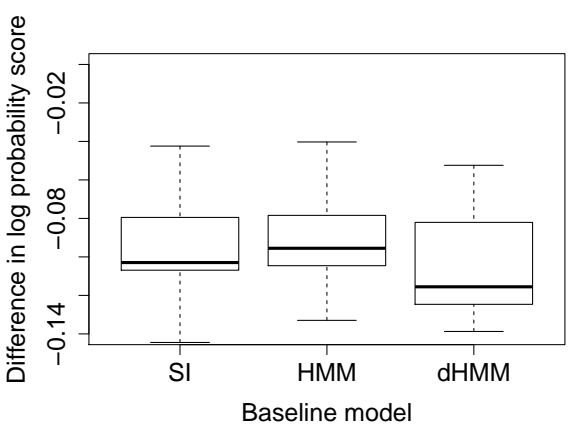

(b)

Figure S.8: Each boxplot shows the log-probability scores, across the validation sets, for a different model. The $\mathrm{y}$-axis is defined as the log-probability score for (a) other numbers of changepoints and (b) other baseline models, minus the log-probability score for the model with two changepoints.

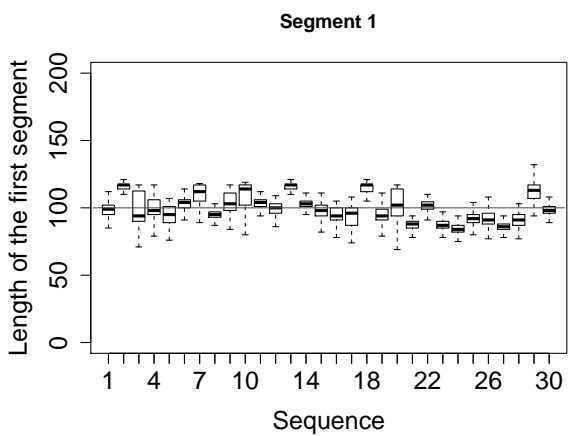

(a)

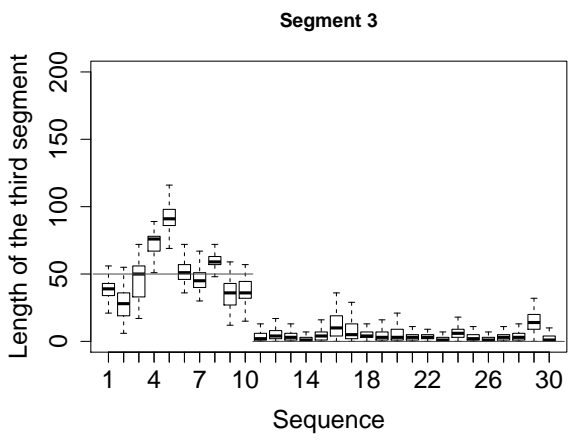

(c)

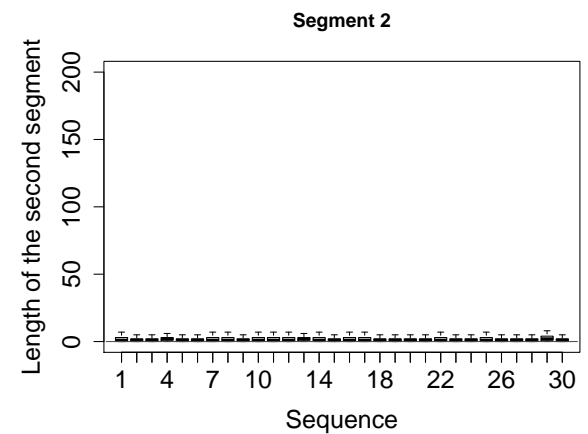

(b)

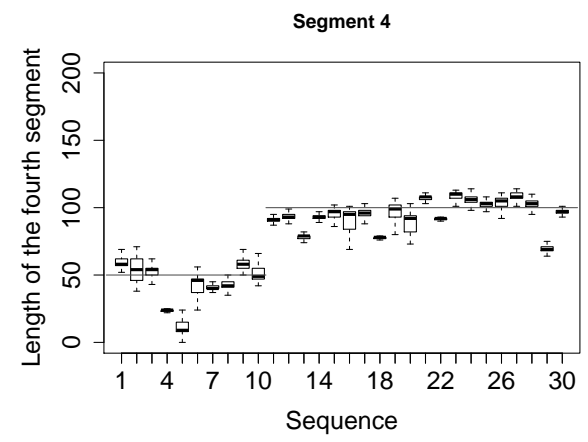

(d)

Figure S.9: Distribution of the length of each segments in each sequence in the $K=3$ solution. a)-d) are segments 1 to 4 respectively. Each sequence has a box plot for the posterior estimate of the length of its segment. For simplicity we show the expected length of each segment as a solid gray line, noting that for each simulated sequence the actual segment length will vary around this expected length.

\section{S.3.3 Synthetic Data Example: Scenario 4 And 5}

In this section we briefly present an experiment that illustrates the performance of our procedure when the number of possible categories for $y_{j}$ increases, i.e. the dimension of $Q^{(i)}$ increases. In 


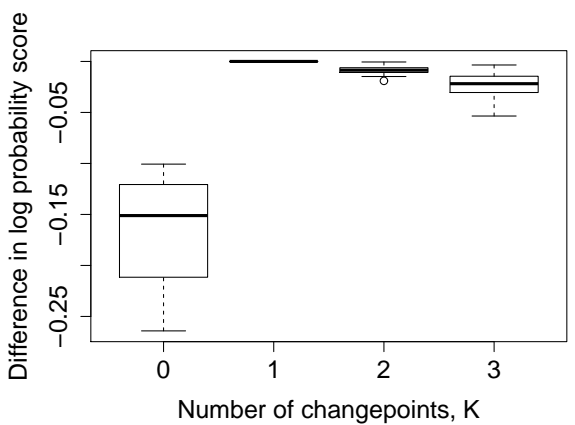

(a)

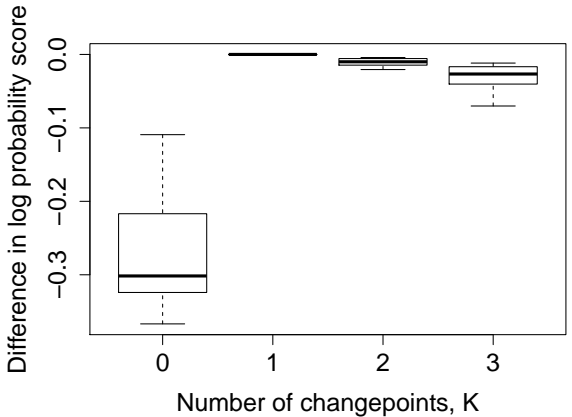

(b)

Figure S.10: Each boxplot shows the log-probability scores, across the validation sets, for a different model. The y-axis is defined as the log-probability score for (a) other numbers of changepoints for scenario 4 and (b) other numbers of changepoints for scenario 5.

particular we keep the changepoints simulated for the 10 sequences in Section 4.1 in the paper, but simulate instead $\mathbf{y}$, for scenario 4 , by using the following transition matrices

$$
\boldsymbol{Q}^{(1)}=\left[\begin{array}{lll}
0.8 & 0.1 & 0.1 \\
0.1 & 0.8 & 0.1 \\
0.1 & 0.1 & 0.8
\end{array}\right], \boldsymbol{Q}^{(2)}=\left[\begin{array}{ccc}
0.333 & 0.333 & 0.333 \\
0.350 & 0.300 & 0.350 \\
0.375 & 0.375 & 0.250
\end{array}\right]
$$

and for scenario 5 the matrices

$$
\boldsymbol{Q}^{(1)}=\left[\begin{array}{llll}
0.800 & 0.033 & 0.033 & 0.033 \\
0.033 & 0.800 & 0.033 & 0.033 \\
0.033 & 0.033 & 0.800 & 0.033 \\
0.033 & 0.033 & 0.033 & 0.800
\end{array}\right], \boldsymbol{Q}^{(2)}=\left[\begin{array}{llll}
0.250 & 0.250 & 0.250 & 0.250 \\
0.267 & 0.200 & 0.267 & 0.267 \\
0.283 & 0.283 & 0.150 & 0.283 \\
0.300 & 0.300 & 0.300 & 0.100
\end{array}\right]
$$

which are chosen to somewhat imitate the transition matrices in Section 4.1 but now with 3 and 4 categories, respectively. Figure $\underline{\mathbf{S . 1 0}}$ shows the result when running our cross-validation procedure on these two data sets. In both of these two multi-category examples we are able to detect the correct number of changepoints. We next investigate the distribution of the position of the changepoints under these two scenarios, see Figure S.11. Comparing these results to Figure 3 in the paper, we see that the uncertainty regarding the position of the changepoints increases due to less data, although the results are still relatively accurate. Similar results can be seen in the estimates for the model parameters $\boldsymbol{Q}^{(1)}, \boldsymbol{Q}^{(2)}, r_{1}$ and $b_{1}$ (not shown). 


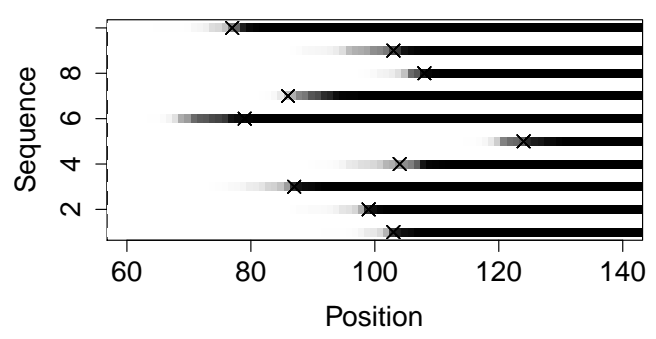

(a)

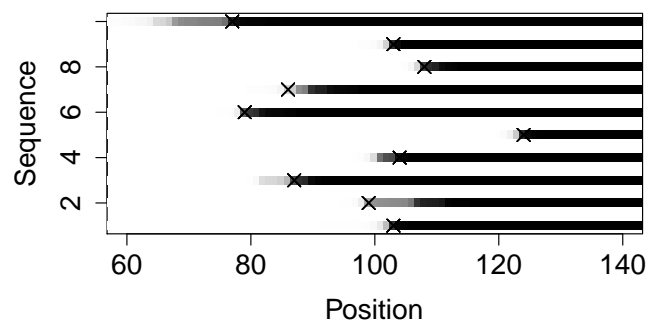

(c)

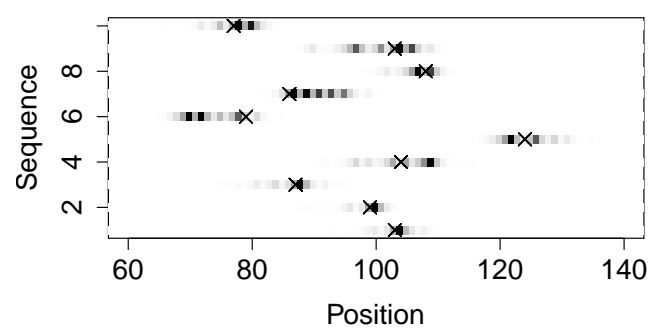

(b)

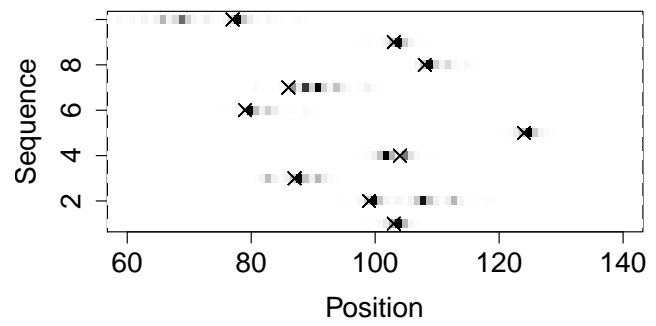

(d)

Figure S.11: The estimated marginal probability distribution of (a) the classification of each node to segment 2 (probability 1 is black) for scenario 4 and (b) a particular node being a changepoint for scenario 4, where the gray scale has been adjusted for each sequence so that the node with the maximum probability is black and the node with the minimum probability is white. In (c) and (d) similar results are shown for scenario 5 respectively. 